Electronic Supplementary Material (ESI) for Catalysis Science \& Technology.

This journal is () The Royal Society of Chemistry 2015

Supporting Information

for

\title{
Selective NaOH-catalysed hydration of aromatic nitriles to amides
}

Thibault E. Schmid, Alberto Gómez-Herrera, Olivier Songis, Deborah Sneddon, Antoine Révolte, Fady Nahra and Catherine S. J. Cazin*

EaStCHEM School of Chemistry, University of St Andrews, St Andrews KY16 9ST, U.K. cc111@st-andrews.ac.uk 


\section{Table of Contents}

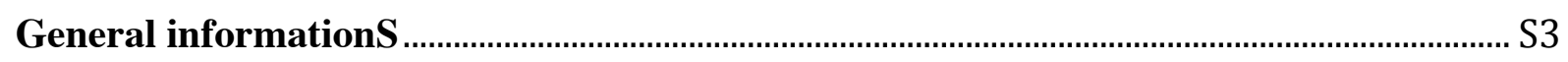

General procedure for the optimization of reaction conditions (Table 1) ...........................S3

General procedure for nitriles hydration (Scheme 1) …….................................................... S3

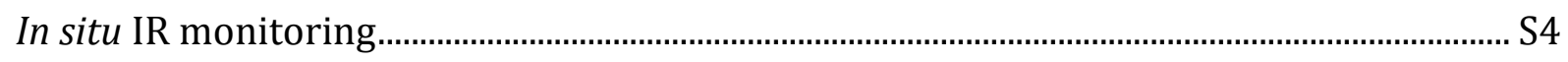

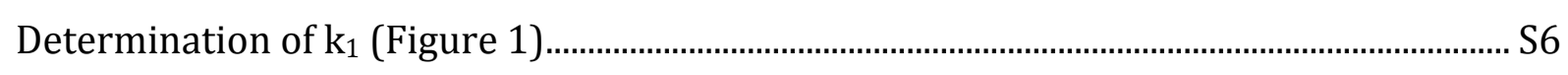

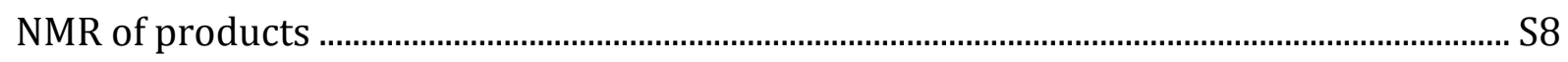

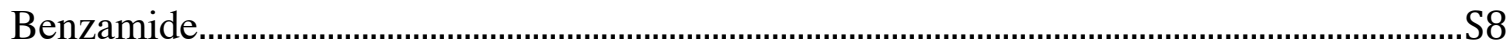

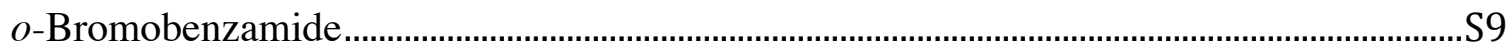

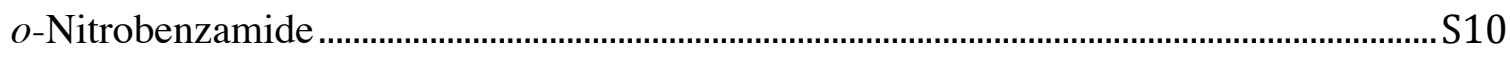

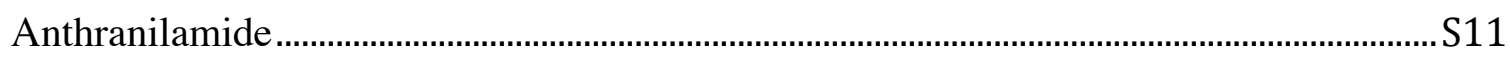

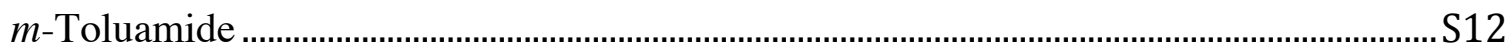

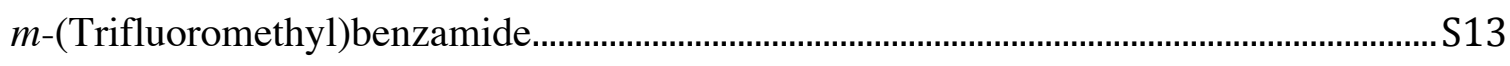

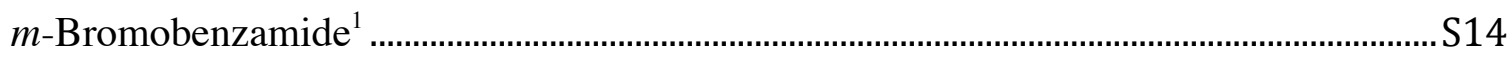

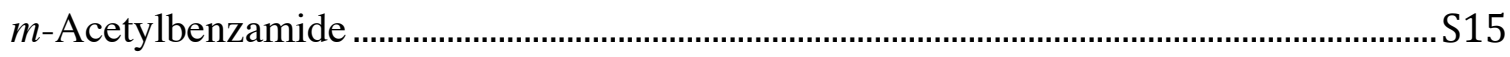

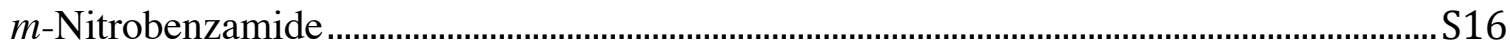

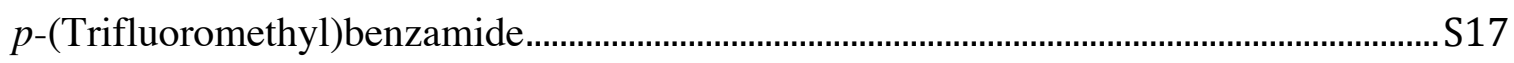

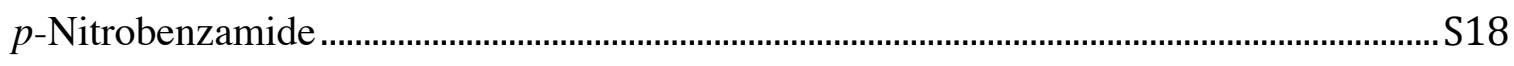

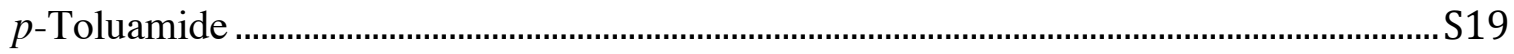

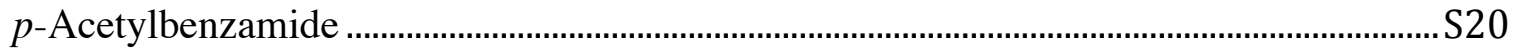

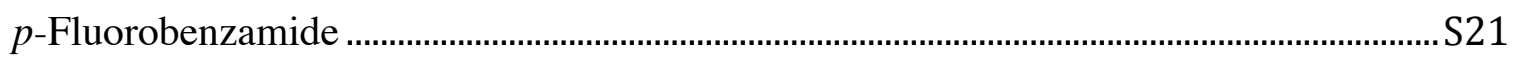

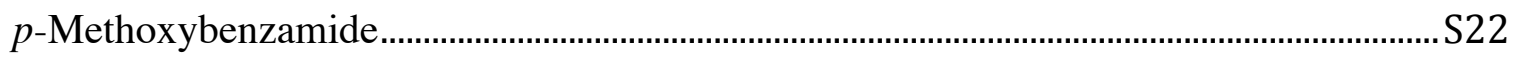

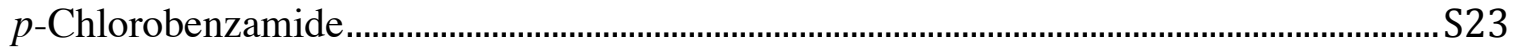

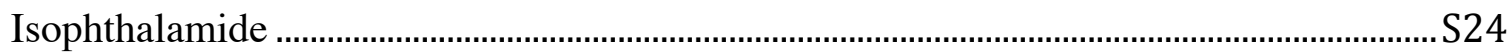

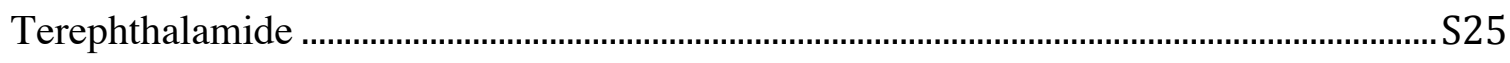

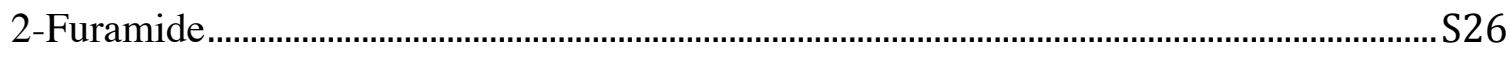

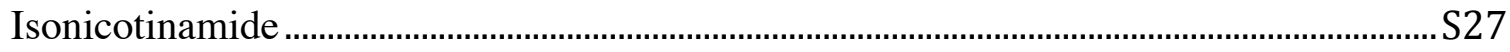

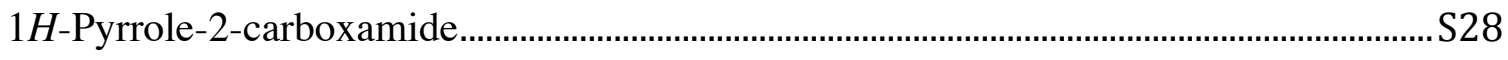

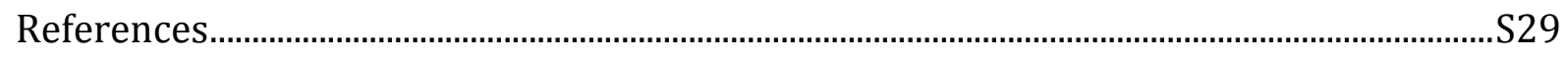




\section{General information}

All reactions were carried out in air. Flash column chromatography purifications were performed on silica gel 60 (230-400 mesh). ${ }^{1} \mathrm{H}$ and ${ }^{13} \mathrm{C}-\left\{{ }^{1} \mathrm{H}\right\}$ Nuclear Magnetic Resonance (NMR) spectra were recorded on a Bruker AC300 or on a Bruker Advance 400 Ultrashield spectrometer at $298 \mathrm{~K}$, gas chromatography (GC) analyses were performed on an Agilent 7890A apparatus equipped with a flame ionisation detector and a (5\%-Phenyl)methylpolysiloxane column. IR measurements were performed on a ReactIR A15 system (Mettler Toledo Autochem) with a DiComp AgX FiberConduit probe (9.5 mm). All glassware was dried in an oven at $80{ }^{\circ} \mathrm{C}$ prior to use. All reagents (unless otherwise noted) were purchased and used without further purification. Solvents were purchased and used as received.

\section{General procedure for the optimization of reaction conditions (Table} 1)

A screwcap vial fitted with a septum and equipped with a stirring bar was charged with benzonitrile $(0.1 \mathrm{~mL}, 1 \mathrm{mmol})$, the required amount of base, and the solvent $(0.5 \mathrm{~mL})$. The reaction mixture was stirred at $90{ }^{\circ} \mathrm{C}$ or $110{ }^{\circ} \mathrm{C}$ (external temperature) for 17 hours. A saturated solution of sodium chloride $(5 \mathrm{~mL})$ and EtOAc $(5 \mathrm{~mL})$ were added to the reaction mixture. The two layers were separated and the aqueous phase was extracted with EtOAc $(5 \times 5 \mathrm{~mL})$. The aqueous phase was acidified by addition of an $\mathrm{HCl}$ aqueous solution $(1 \mathrm{M}, 10$ $\mathrm{mL})$, and the resulting suspension was extracted with EtOAc $(5 \times 5 \mathrm{~mL})$. All organic extracts were combined, dried over $\mathrm{MgSO}_{4}$, filtered, and concentrated under vacuum. The resulting mixture was diluted in acetone $(10 \mathrm{~mL})$, and a standard solution of mesitylene $(0.5 \mathrm{M}$ in acetone, $1 \mathrm{~mL}$ ) was added for GC analysis.

\section{General procedure for nitriles hydration (Scheme 1)}

A screwcap-vial fitted with a septum and equipped with a stirring bar was charged with the nitrile $(1 \mathrm{mmol}), \mathrm{NaOH}(0.1 \mathrm{mmol})$ and the solvent $(0.5 \mathrm{~mL})$. The reaction mixture was stirred at $90{ }^{\circ} \mathrm{C}$ (external temperature) for 17 hours.

\section{Workup A}

A saturated solution of sodium chloride $(5 \mathrm{~mL})$ and EtOAc $(5 \mathrm{~mL})$ were added to the reaction mixture. The two layers were separated and the aqueous phase was extracted with EtOAc $(5 \times 5 \mathrm{~mL})$. The organic extracts were combined, dried over $\mathrm{MgSO}_{4}$, filtered and concentrated 
under vacuum. If necessary, the resulting solid was purified by flash chromatography using a gradient of pentane/EtOAc $(7: 3$ to $0: 1)$.

\section{Workup B}

The reaction mixture was cooled down to $4{ }^{\circ} \mathrm{C}$ for $24 \mathrm{~h}$. The resulting precipitate was collected by filtration and washed with water $(4 \mathrm{~mL})$ and acetone $(4 \mathrm{~mL})$, and further dried at $80{ }^{\circ} \mathrm{C}$ for $5 \mathrm{~h}$.

\section{In situ IR monitoring}

Sodium benzoate and sodium acetate were freshly prepared by boiling benzoic acid or glacial acetic acid with an aqueous $\mathrm{NaOH}$ solution until dryness. All other substrates were commercially available. $2 \mathrm{M}$ solutions $\left(15 \mathrm{~mL}, \mathrm{EtOH} / \mathrm{H}_{2} \mathrm{O}, 7: 3\right)$ were prepared for the IR spectra library. All spectra were collected at $80^{\circ} \mathrm{C}$ in air.

A $25 \mathrm{~mL}$ two-necked round-bottomed flask was charged with the substrate $(24 \mathrm{mmol})$ and EtOH $/ \mathrm{H}_{2} \mathrm{O}(7: 3 \mathrm{v} / \mathrm{v}, 12 \mathrm{~mL})$. The reaction mixture was warmed to $90{ }^{\circ} \mathrm{C}$ (external temperature, internal temperature: $83-84{ }^{\circ} \mathrm{C}$ ), and IR monitoring was started. The catalyst, $\mathrm{NaOH}(10 \mathrm{~mol} \%)$ was then added, and the reaction evolution was monitored.
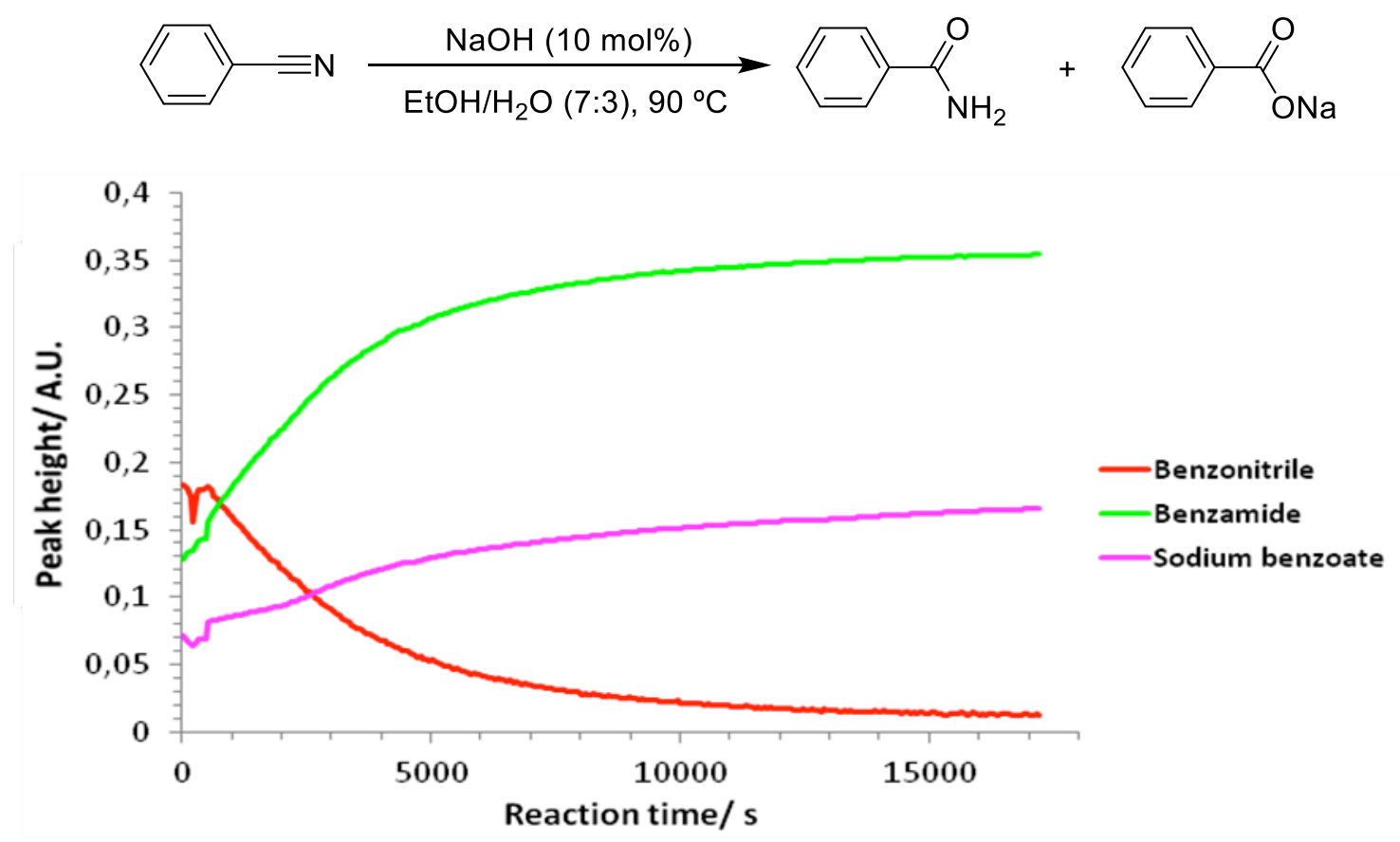

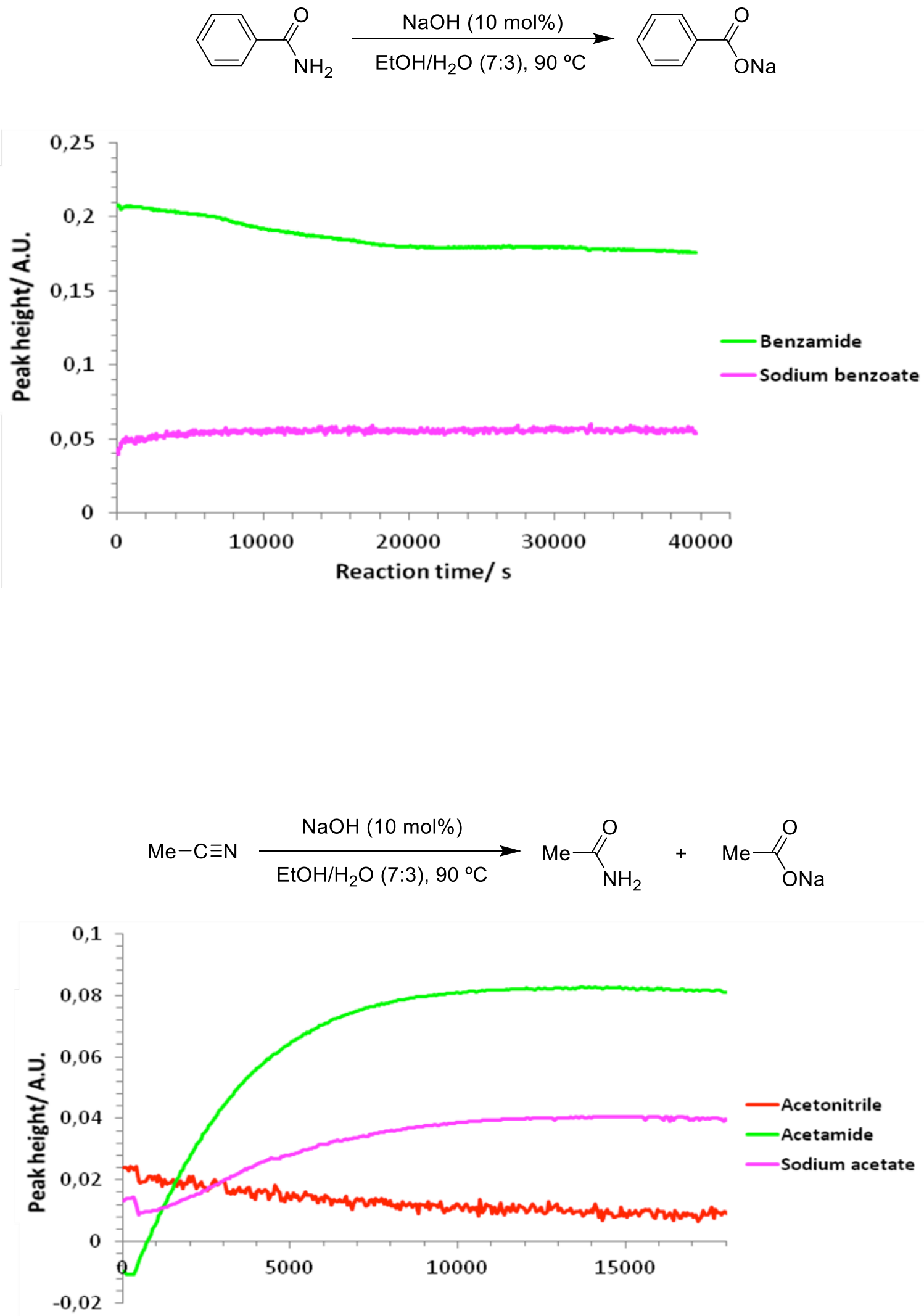

Reaction time/s 


\section{Determination of $\mathbf{k}_{\mathbf{1}}$ (Figure 1 )}

The validity of the Beer- Lambert law was considered for the presented reactive system.

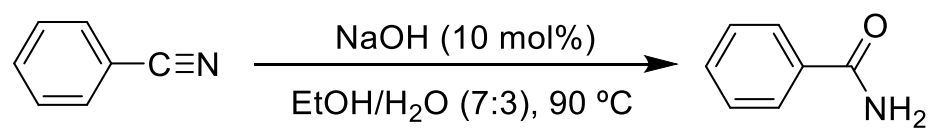

Differential rate equation: $\quad \mathrm{v}=\mathrm{k}_{1}[\mathrm{PhCN}]\left[\mathrm{OH}^{-}\right]$

Catalytic base $\rightarrow \mathrm{v}=\mathrm{k}^{\prime}{ }_{1}[\mathrm{PhCN}]$

Integrated rate equation: $\operatorname{Ln}[\mathrm{PhCN}]=\mathrm{Ln}[\mathrm{PhCN}]_{0}-\mathrm{k}_{1}{ }_{1} \mathrm{t}$

COMPLETE EXPERIMENTAL PLOT

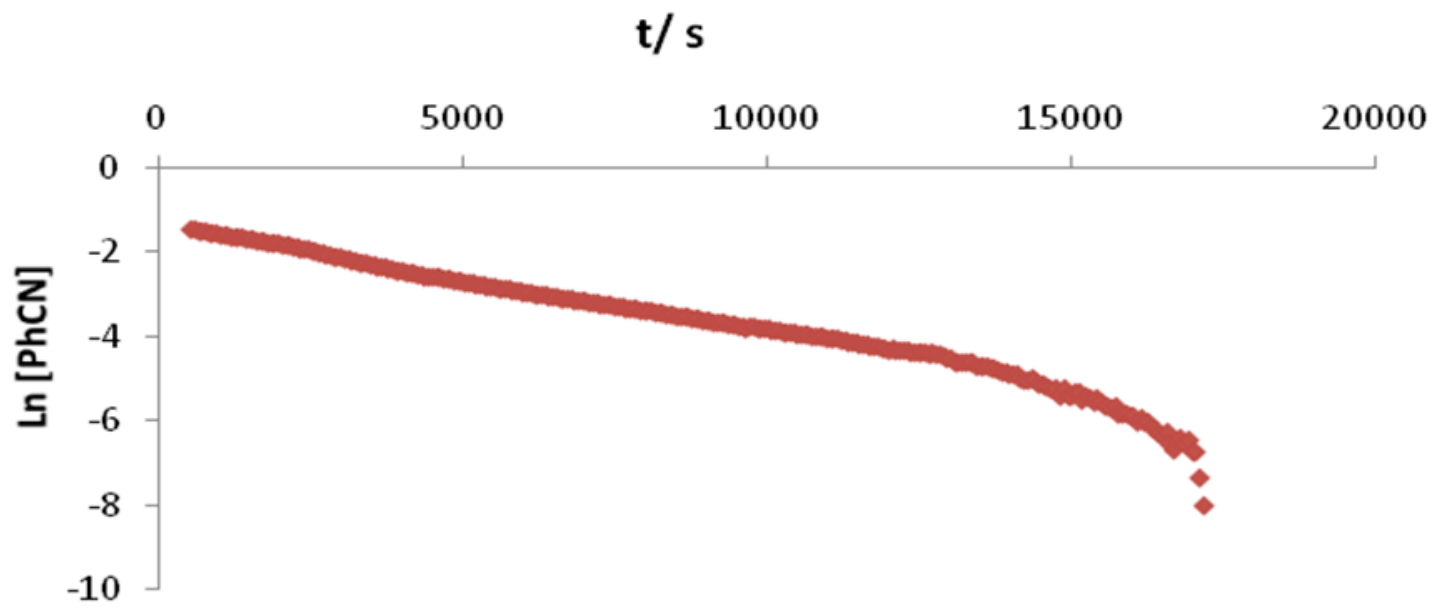

REFINED EXPERIMENTAL PLOT ${ }^{\mathrm{a}}$

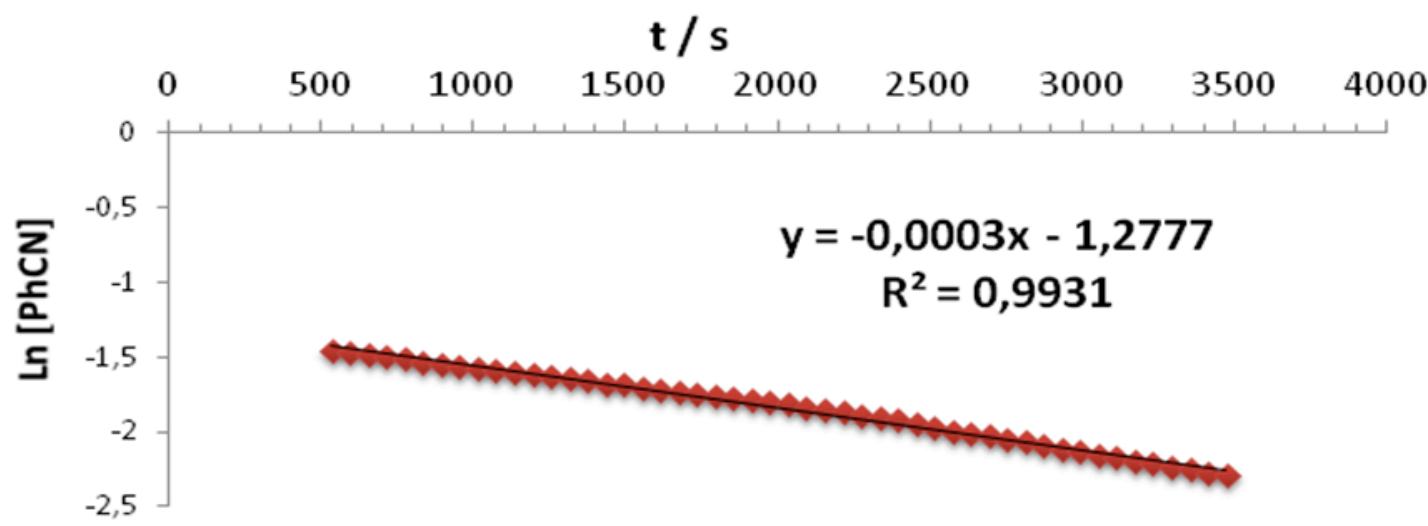

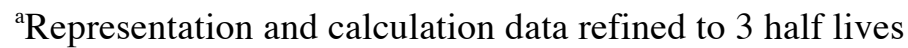

$$
\mathrm{Ln}[\mathrm{PhCN}]=\mathrm{Ln}[\mathrm{PhCN}]_{0}-\mathrm{k}_{1}{ }_{1} \mathrm{t}
$$


1. $\mathrm{k}_{1}{ }_{1}=3 \cdot 10^{-4} \mathrm{~s}^{-1}$

2. $\mathrm{t}_{1 / 2}=(\operatorname{Ln} 2) / \mathrm{k}^{\prime}{ }_{1} \approx 39 \mathrm{~min}$

3. $\mathrm{Ln}[\mathrm{PhCN}]_{0}=-1.2777 \quad ; \quad[\mathrm{PhCN}]_{0}=0.28 \mathrm{M} ;\left[\mathrm{OH}^{-}\right]_{0}=\left[\mathrm{OH}^{-}\right]=0.1 \times[\mathrm{PhCN}]_{0}=0.028$ $\mathrm{M}$

4. $\mathrm{k}^{\prime}{ }_{1}=\mathrm{k}_{1}\left[\mathrm{OH}^{-}\right] \rightarrow \mathrm{k}_{1}=1.1 \cdot 10^{-2} \mathrm{M}^{-1} \cdot \mathrm{s}^{-1}$ 


\section{NMR of products}

\section{Benzamide $^{1}$}

The general procedure afforded the title compound (workup A) as a colourless solid (99 mg, $82 \%)$.

${ }^{1} \mathrm{H}$ NMR $\left(400 \mathrm{MHz}, 298 \mathrm{~K}, \mathrm{DMSO}-d_{6}\right): \delta(\mathrm{ppm})=7.36$ (br. s, $\left.1 \mathrm{H}, \mathrm{NH}\right), 7.42-7.47(\mathrm{~m}, 2 \mathrm{H}$, $\left.\mathrm{CH}_{\mathrm{Ar}}\right), 7.50-7.54\left(\mathrm{~m}, 1 \mathrm{H}, \mathrm{CH}_{\mathrm{Ar}}\right), 7.85-7.89\left(\mathrm{~m}, 2 \mathrm{H}, \mathrm{CH}_{\mathrm{Ar}}\right), 7.96$ (br. s, $\left.1 \mathrm{H}, \mathrm{NH}\right)$.

${ }^{13} \mathrm{C}-\left\{{ }^{1} \mathrm{H}\right\}$ NMR $\left(100.62 \mathrm{MHz}, 298 \mathrm{~K}, \mathrm{DMSO}-d_{6}\right): \delta(\mathrm{ppm})=127.4\left(\mathrm{~s}, \mathrm{CH}_{\mathrm{Ar}}\right), 128.2\left(\mathrm{~s}, \mathrm{CH}_{\mathrm{Ar}}\right)$, $131.2\left(\mathrm{~s}, \mathrm{CH}_{\mathrm{Ar}}\right), 134.3\left(\mathrm{~s}, \mathrm{C}_{\mathrm{Ar}}\right), 167.9\left(\mathrm{~s}, \mathrm{CONH}_{2}\right)$.
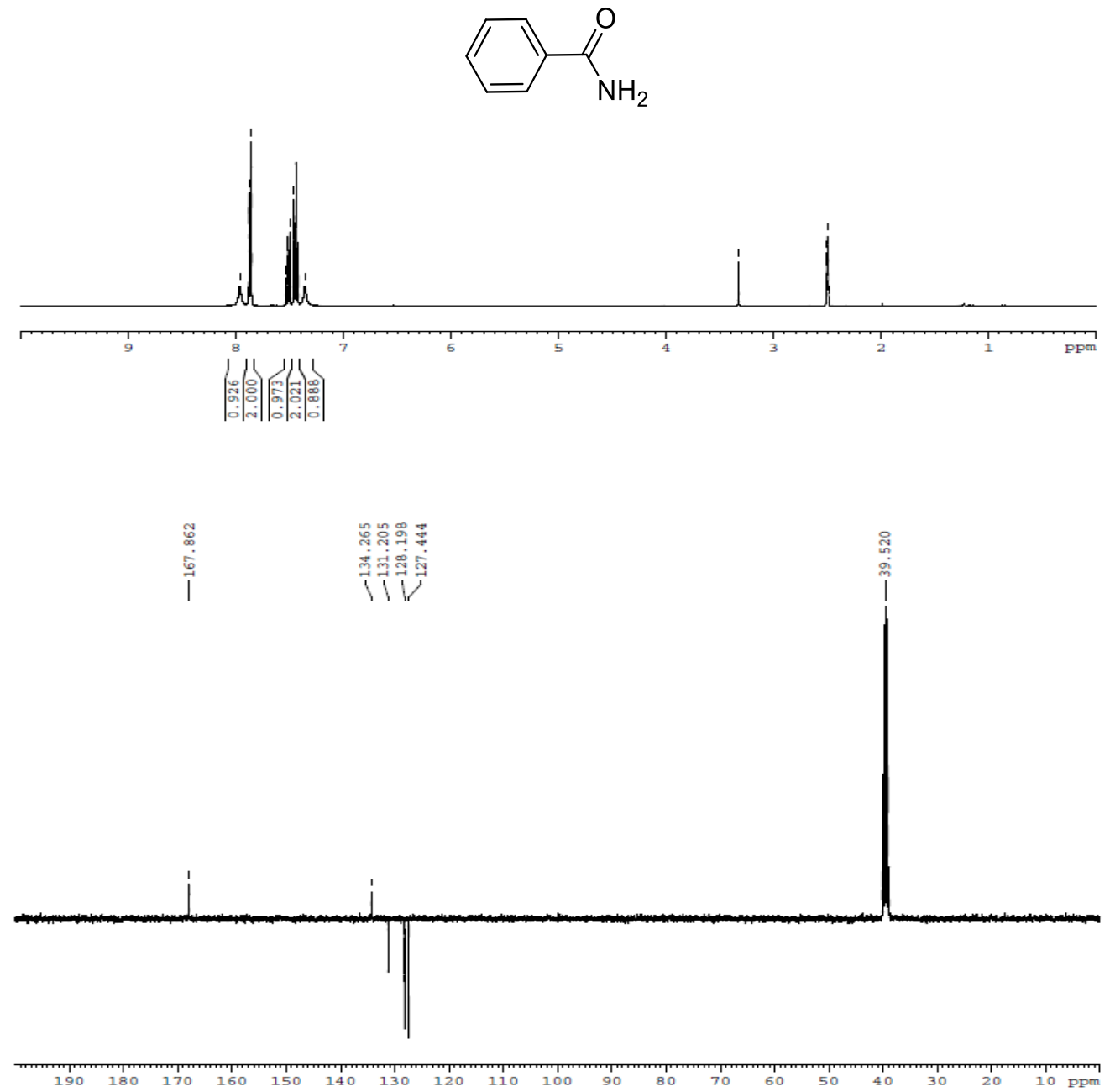


\section{$o$-Bromobenzamide ${ }^{1}$}

The general procedure afforded the title compound (workup A) as a colourless solid (174 mg, $87 \%)$.

${ }^{1} \mathrm{H}$ NMR (400 MHz, 298K, DMSO- $\left.d_{6}\right): \delta(\mathrm{ppm})=7.30-7.35\left(\mathrm{~m}, 1 \mathrm{H}, \mathrm{CH}_{\mathrm{Ar}}\right), 7.39-7.41(\mathrm{~m}$, $2 \mathrm{H}, \mathrm{CH}_{\mathrm{Ar}}$ ), 7.56 (br. s, $\left.1 \mathrm{H}, \mathrm{NH}\right), 7.62-7.64\left(\mathrm{~m}, 1 \mathrm{H}, \mathrm{CH}_{\mathrm{Ar}}\right), 7.85$ (br. s, $\left.1 \mathrm{H}, \mathrm{NH}\right)$.

${ }^{13} \mathrm{C}-\left\{{ }^{1} \mathrm{H}\right\}$ NMR $\left(100.62 \mathrm{MHz}, 298 \mathrm{~K}, \mathrm{DMSO}-d_{6}\right): \delta(\mathrm{ppm})=118.6\left(\mathrm{~s}, \mathrm{C}_{\mathrm{Ar}}\right), 127.5\left(\mathrm{~s}, \mathrm{CH}_{\mathrm{Ar}}\right)$, $128.5\left(\mathrm{~s}, \mathrm{CH}_{\mathrm{Ar}}\right), 130.6\left(\mathrm{~s}, \mathrm{CH}_{\mathrm{Ar}}\right), 132.7\left(\mathrm{~s}, \mathrm{CH}_{\mathrm{Ar}}\right), 139.4\left(\mathrm{~s}, \mathrm{C}_{\mathrm{Ar}}\right), 169.0\left(\mathrm{~s}, \mathrm{CONH}_{2}\right)$.

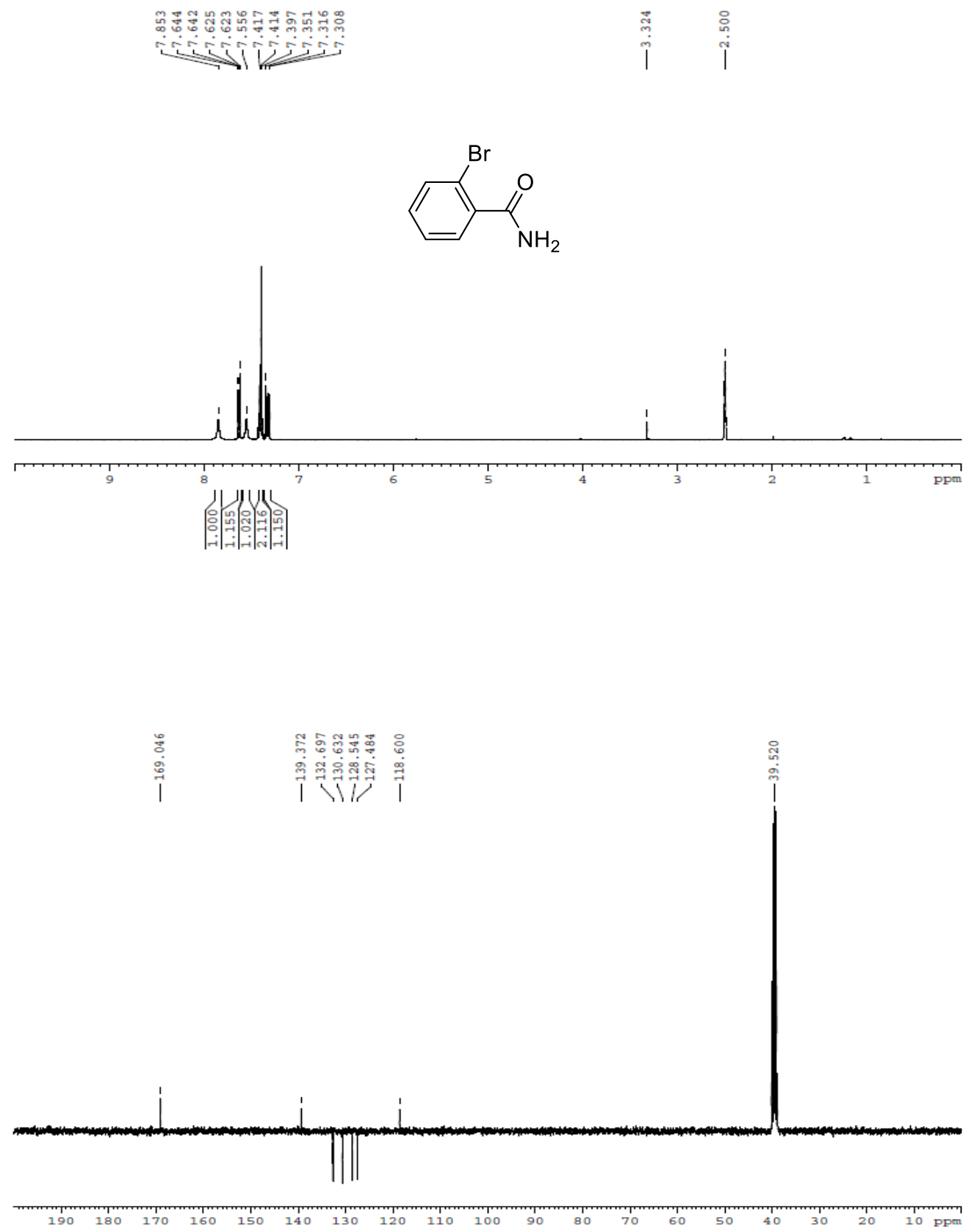




\section{$o$-Nitrobenzamide ${ }^{1}$}

The general procedure afforded the title compound (workup A followed by flash chromatography) as a colourless solid (113 mg, 68\%).

${ }^{1} \mathrm{H}$ NMR (400 MHz, 298K, DMSO- $\left.d_{6}\right): \delta(\mathrm{ppm})=7.61-7.72\left(\mathrm{~m}, 3 \mathrm{H}, \mathrm{C} H_{\mathrm{Ar}}+\mathrm{NH}\right), 7.76(\mathrm{td}$, $\left.{ }^{3} J_{\mathrm{H}-\mathrm{H}}=8.0 \mathrm{~Hz},{ }^{4} J_{\mathrm{H}-\mathrm{H}}=1.2 \mathrm{~Hz}, 1 \mathrm{H}, \mathrm{CH}_{\mathrm{Ar}}\right), 7.99\left(\mathrm{dd},{ }^{3} J_{\mathrm{H}-\mathrm{H}}=8.0 \mathrm{~Hz},{ }^{4} J_{\mathrm{H}-\mathrm{H}}=1.2 \mathrm{~Hz}, 1 \mathrm{H}, \mathrm{CH}_{\mathrm{Ar}}\right)$, 8.14 (br. s, $1 \mathrm{H}, \mathrm{NH}$ ).

${ }^{13} \mathrm{C}-\left\{{ }^{1} \mathrm{H}\right\}$ NMR $\left(100.62 \mathrm{MHz}, 298 \mathrm{~K}, \mathrm{DMSO}-d_{6}\right): \delta(\mathrm{ppm})=123.9\left(\mathrm{~s}, \mathrm{CH}_{\mathrm{Ar}}\right), 128.8\left(\mathrm{~s}, \mathrm{CH}_{\mathrm{Ar}}\right)$, $130.6\left(\mathrm{~s}, \mathrm{CH}_{\mathrm{Ar}}\right), 132.6\left(\mathrm{~s}, \mathrm{C}_{\mathrm{Ar}}\right), 133.3\left(\mathrm{~s}, \mathrm{CH}_{\mathrm{Ar}}\right), 147.2\left(\mathrm{~s}, \mathrm{C}_{\mathrm{Ar}}\right), 167.1\left(\mathrm{~s}, \mathrm{CONH}_{2}\right)$.

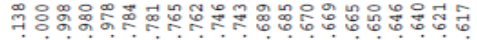

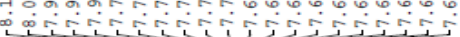

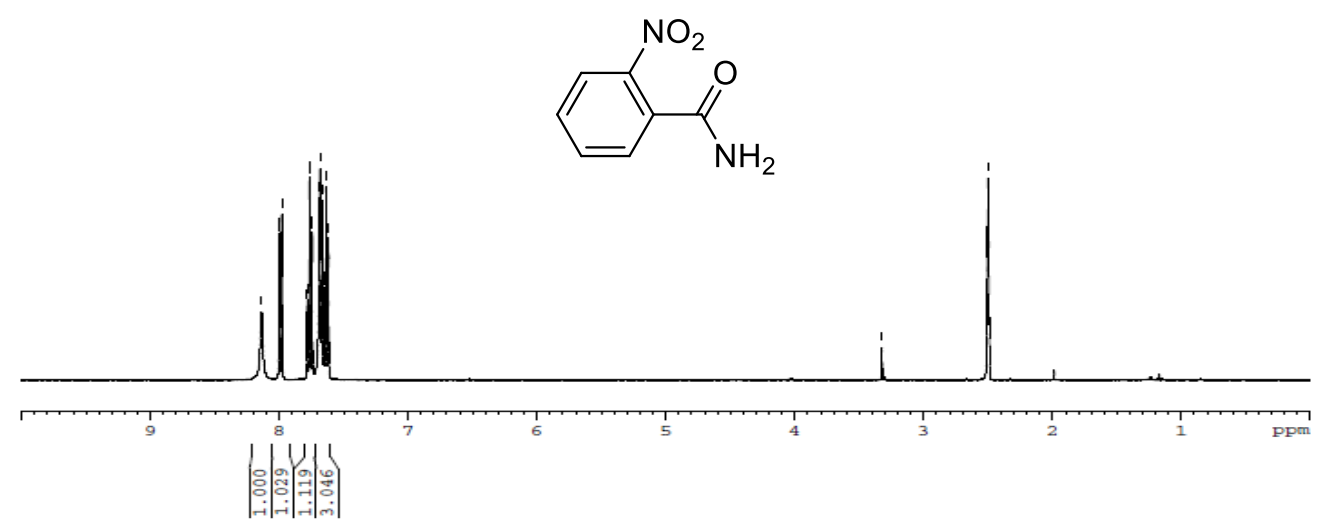

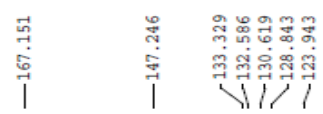

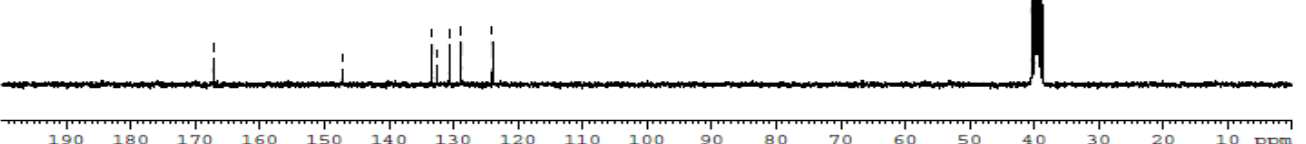




\section{Anthranilamide ${ }^{2}$}

The general procedure afforded the title compound by using (workup A followed by flash chromatography) as a colourless solid (86 mg, 63\%).

${ }^{1} \mathrm{H}$ NMR (400 MHz, 298K, DMSO-d 6$): \delta(\mathrm{ppm})=6.44-6.49\left(\mathrm{~m}, 1 \mathrm{H}, \mathrm{CH}_{\mathrm{Ar}}\right), 6.55(\mathrm{~s}, 2 \mathrm{H}$, $\left.\mathrm{NH}_{2}\right), 6.67\left(\mathrm{dd},{ }^{3} J_{\mathrm{H}-\mathrm{H}}=8.0 \mathrm{~Hz},{ }^{4} J_{\mathrm{H}-\mathrm{H}}=1.2 \mathrm{~Hz}, 1 \mathrm{H}, \mathrm{CH}_{\mathrm{Ar}}\right), 7.05$ (br. s, $\left.1 \mathrm{H}, \mathrm{NH}\right), 7.09-7.15(\mathrm{~m}$, $\left.1 \mathrm{H}, \mathrm{CH}_{\mathrm{Ar}}\right), 7.52\left(\mathrm{dd},{ }^{3} J_{\mathrm{H}-\mathrm{H}}=8.0 \mathrm{~Hz},{ }^{4} J_{\mathrm{H}-\mathrm{H}}=1.2 \mathrm{~Hz}, 1 \mathrm{H}, \mathrm{CH}_{\mathrm{Ar}}\right), 7.71$ (br. s, $\left.1 \mathrm{H}, \mathrm{NH}\right)$.

${ }^{13} \mathrm{C}-\left\{{ }^{1} \mathrm{H}\right\}$ NMR $\left(100.62 \mathrm{MHz}, 298 \mathrm{~K}, \mathrm{DMSO}-d_{6}\right): \delta(\mathrm{ppm})=113.7\left(\mathrm{~s}, \mathrm{C}_{\mathrm{Ar}}\right), 114.4\left(\mathrm{~s}, \mathrm{CH}_{\mathrm{Ar}}\right)$, $116.4\left(\mathrm{~s}, \mathrm{CH}_{\mathrm{Ar}}\right), 128.8\left(\mathrm{~s}, \mathrm{CH}_{\mathrm{Ar}}\right), 131.9\left(\mathrm{~s}, \mathrm{CH}_{\mathrm{Ar}}\right), 150.2\left(\mathrm{~s}, \mathrm{C}_{\mathrm{Ar}}\right), 171.3\left(\mathrm{~s}, \mathrm{CONH}_{2}\right)$.
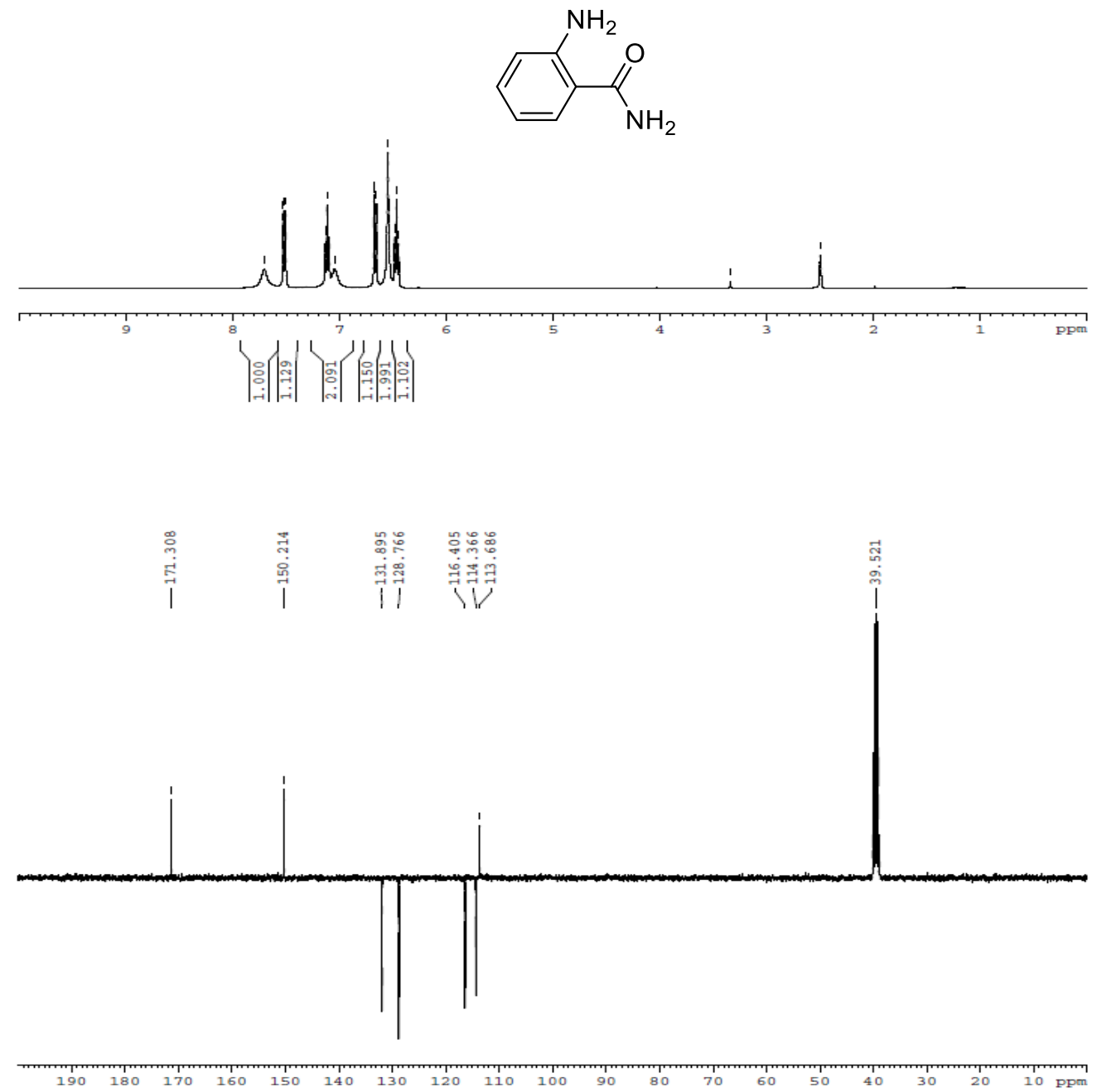


\section{$m$-Toluamide ${ }^{1}$}

The general procedure afforded the title compound (workup A) as a colourless solid (112 mg, $83 \%)$.

${ }^{1} \mathrm{H}$ NMR (400 MHz, 298K, DMSO- $\left.d_{6}\right): \delta(\mathrm{ppm})=2.34\left(\mathrm{~s}, 3 \mathrm{H}, \mathrm{CH}_{3}\right), 7.32\left(\mathrm{~d},{ }^{3} J_{\mathrm{H}-\mathrm{H}}=4.4 \mathrm{~Hz}\right.$, $\left.3 \mathrm{H}, \mathrm{CH}_{\mathrm{Ar}}\right), 7.64-7.69\left(\mathrm{~m}, 1 \mathrm{H}, \mathrm{CH}_{\mathrm{Ar}}\right), 7.70(\mathrm{~s}, 1 \mathrm{H}, \mathrm{NH}), 7.92$ (br. s, 1H, NH).

${ }^{13} \mathrm{C}-\left\{{ }^{1} \mathrm{H}\right\}$ NMR $\left(100.62 \mathrm{MHz}, 298 \mathrm{~K}, \mathrm{DMSO}-d_{6}\right): \delta(\mathrm{ppm})=21.0\left(\mathrm{CH}_{3}\right), 124.6\left(\mathrm{~s}, \mathrm{CH}_{\mathrm{Ar}}\right)$, $128.1\left(\mathrm{~s}, \mathrm{CH}_{\mathrm{Ar}}\right), 131.8\left(\mathrm{~s}, \mathrm{CH}_{\mathrm{Ar}}\right), 134.3\left(\mathrm{~s}, \mathrm{C}_{\mathrm{Ar}}\right), 137.4\left(\mathrm{~s}, \mathrm{C}_{\mathrm{Ar}}\right), 168.0\left(\mathrm{~s}, \mathrm{CONH}_{2}\right)$.
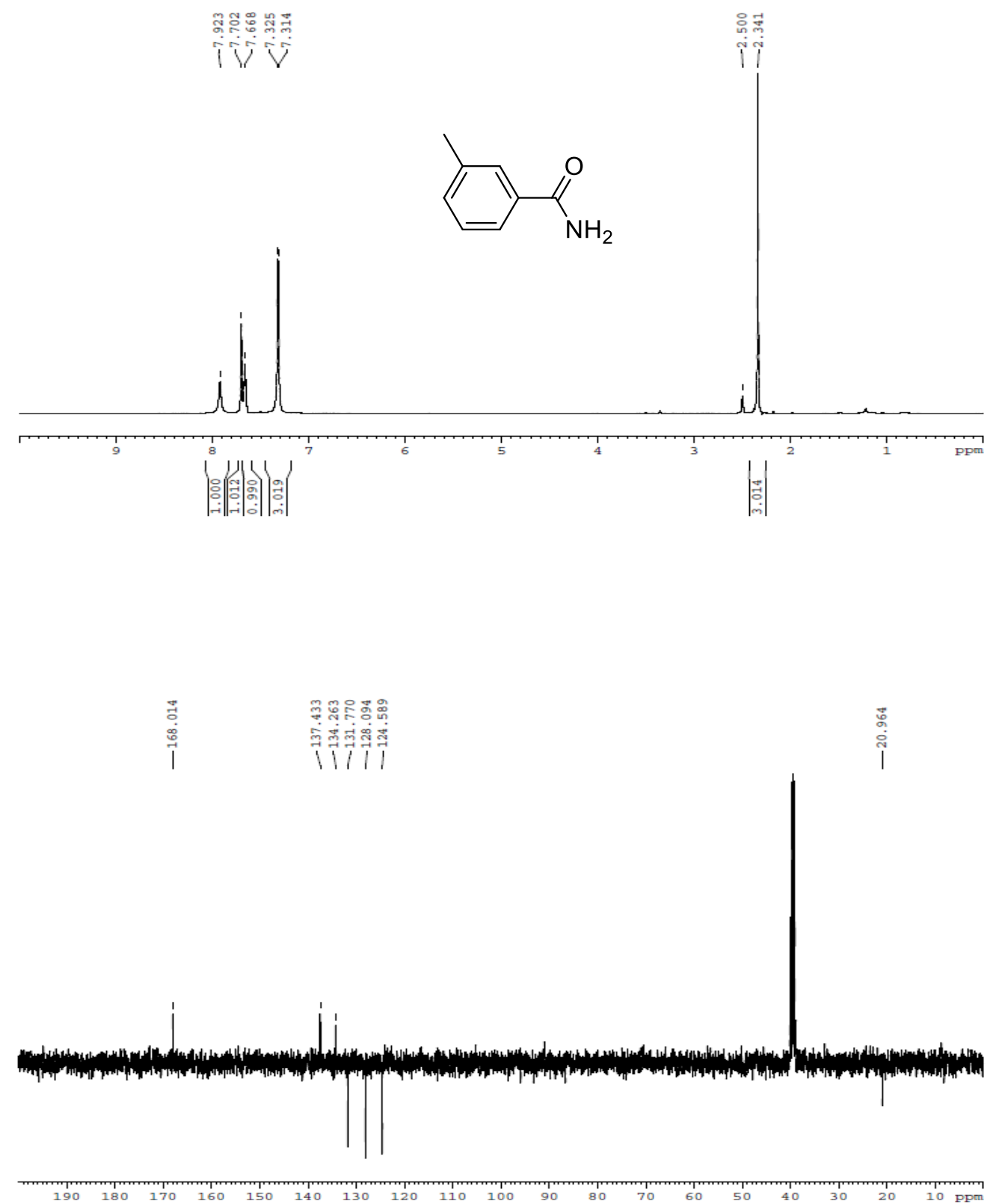


\section{$m$-(Trifluoromethyl)benzamide ${ }^{3}$}

The general procedure afforded the title compound (workup A) as a colourless solid (180 mg, $95 \%)$.

${ }^{1} \mathrm{H}$ NMR $\left(400 \mathrm{MHz}, 298 \mathrm{~K}, \mathrm{DMSO}-d_{6}\right): \delta(\mathrm{ppm})=7.63$ (br. s, $\left.1 \mathrm{H}, \mathrm{NH}\right), 7.71\left(\mathrm{dd},{ }^{3} J_{\mathrm{H}-\mathrm{H}}=8.8\right.$ $\left.\mathrm{Hz},{ }^{3} J_{\mathrm{H}-\mathrm{H}}=7.8 \mathrm{~Hz}, 1 \mathrm{H}, \mathrm{CH}_{\mathrm{Ar}}\right), 7.89\left(\mathrm{~d},{ }^{3} J_{\mathrm{H}-\mathrm{H}}=8.0 \mathrm{~Hz}, 1 \mathrm{H}, \mathrm{CH}_{\mathrm{Ar}}\right), 8.18\left(\mathrm{~d},{ }^{3} J_{\mathrm{H}-\mathrm{H}}=8.0 \mathrm{~Hz}, 1 \mathrm{H}\right.$, $\left.\mathrm{CH}_{\mathrm{Ar}}\right), 8.21\left(\mathrm{~s}, 1 \mathrm{H}, \mathrm{CH}_{\mathrm{Ar}}\right), 8.24(\mathrm{~s}, 1 \mathrm{H}, \mathrm{NH})$.

${ }^{13} \mathrm{C}-\left\{{ }^{1} \mathrm{H}\right\}$ NMR $\left(100.62 \mathrm{MHz}, 298 \mathrm{~K}, \mathrm{DMSO}-d_{6}\right): \delta(\mathrm{ppm})=124.0\left(\mathrm{q},{ }^{1} J_{\mathrm{C}-\mathrm{F}}=272.5 \mathrm{~Hz}, \mathrm{CF}_{3}\right)$, $124.1\left(\mathrm{q},{ }^{3} J_{\mathrm{C}-\mathrm{F}}=3.8 \mathrm{~Hz}, \mathrm{CH}_{\mathrm{Ar}}\right), 127.9\left(\mathrm{q},{ }^{3} J_{\mathrm{C}-\mathrm{F}}=3.8 \mathrm{~Hz}, \mathrm{CH}_{\mathrm{Ar}}\right), 129.1\left(\mathrm{q},{ }^{2} J_{\mathrm{C}-\mathrm{F}}=32.0 \mathrm{~Hz}\right.$, $\left.\mathrm{C}_{\mathrm{Ar}}\right), 129.6\left(\mathrm{~s}, \mathrm{CH}_{\mathrm{Ar}}\right), 131.5\left(\mathrm{~s}, \mathrm{CH}_{\mathrm{Ar}}\right), 135.2\left(\mathrm{~s}, \mathrm{C}_{\mathrm{Ar}}\right), 166.4\left(\mathrm{~s}, \mathrm{CONH}_{2}\right)$.

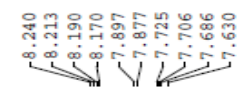
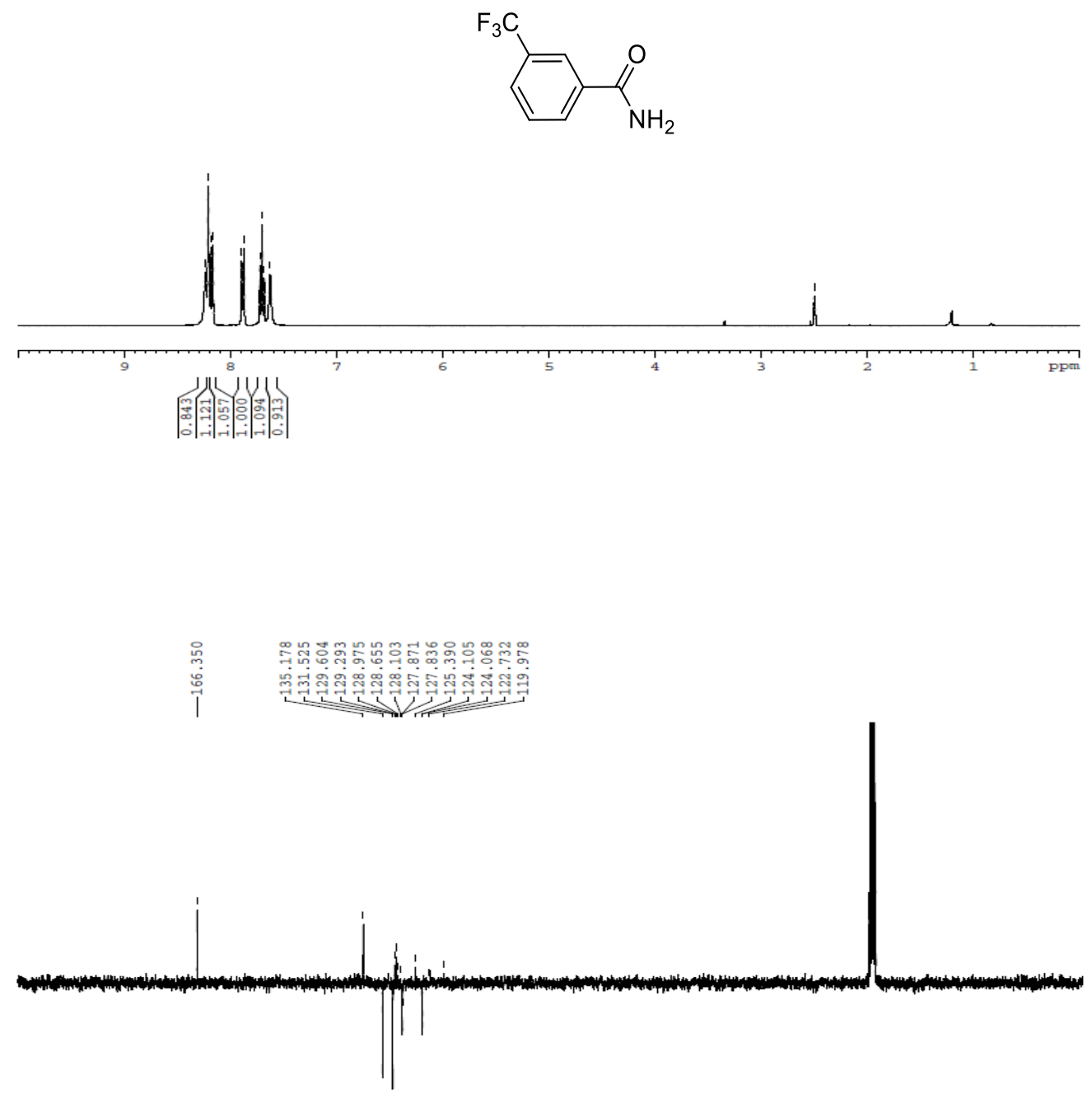

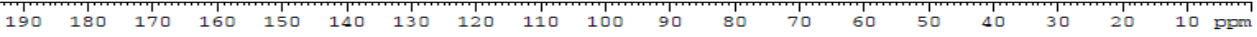




\section{$m$-Bromobenzamide ${ }^{1}$}

The general procedure afforded the title compound (workup A) as a colourless solid (178 mg, $89 \%$ ).

${ }^{1} \mathrm{H}$ NMR $\left(400 \mathrm{MHz}, 298 \mathrm{~K}\right.$, DMSO- $\left.d_{6}\right): \delta(\mathrm{ppm})=7.43\left(\mathrm{t},{ }^{3} J_{\mathrm{H}-\mathrm{H}}=8.0 \mathrm{~Hz}, 1 \mathrm{H}, \mathrm{CH}_{\mathrm{Ar}}\right), 7.52(\mathrm{br}$. $\mathrm{s}, 1 \mathrm{H}, \mathrm{NH}), 7.71-7.74\left(\mathrm{~m}, 1 \mathrm{H}, \mathrm{CH}_{\mathrm{Ar}}\right), 7.85-7.88\left(\mathrm{~m}, 1 \mathrm{H}, \mathrm{CH}_{\mathrm{Ar}}\right), 8.04\left(\mathrm{~d},{ }^{4} J_{\mathrm{H}-\mathrm{H}}=1.2 \mathrm{~Hz}, 1 \mathrm{H}\right.$, $\mathrm{CH}_{\mathrm{Ar}}$ ), 8.08 (br. s, $\left.1 \mathrm{H}, \mathrm{NH}\right)$.

${ }^{13} \mathrm{C}-\left\{{ }^{1} \mathrm{H}\right\}$ NMR $\left(100.62 \mathrm{MHz}, 298 \mathrm{~K}, \mathrm{DMSO}-d_{6}\right): \delta(\mathrm{ppm})=121.6\left(\mathrm{~s}, \mathrm{C}_{\mathrm{Ar}}\right), 126.6\left(\mathrm{~s}, \mathrm{CH}_{\mathrm{Ar}}\right)$, $130.2\left(\mathrm{~s}, \mathrm{CH}_{\mathrm{Ar}}\right), 130.5\left(\mathrm{~s}, \mathrm{CH}_{\mathrm{Ar}}\right), 134.0\left(\mathrm{~s}, \mathrm{CH}_{\mathrm{Ar}}\right), 136.5\left(\mathrm{~s}, \mathrm{C}_{\mathrm{Ar}}\right), 166.3\left(\mathrm{~s}, \mathrm{CONH}_{2}\right)$.

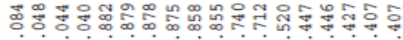

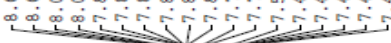
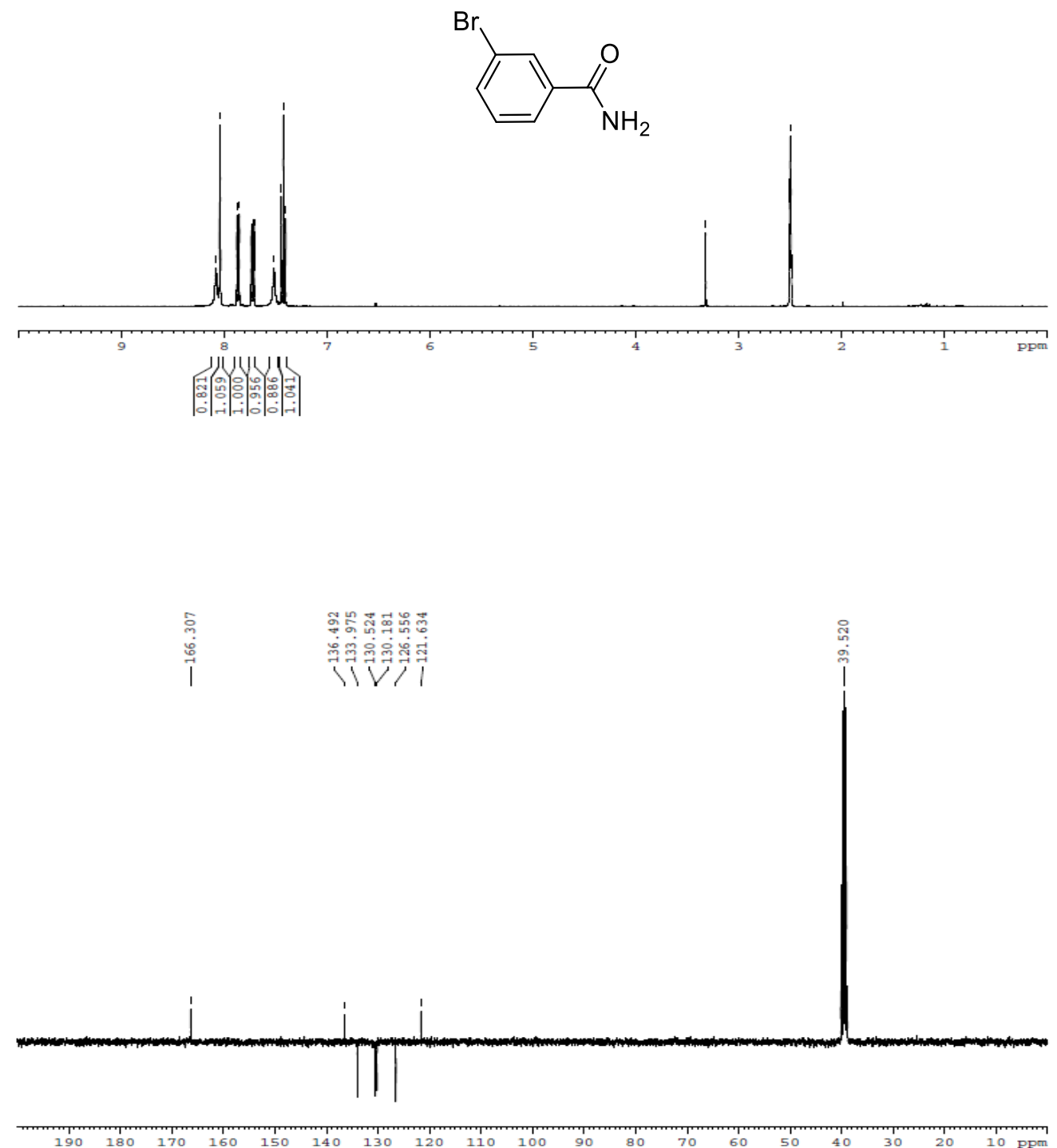


\section{$m$-Acetylbenzamide ${ }^{1}$}

The general procedure afforded the title compound (workup A followed by flash chromatography) as a colourless solid (142 mg, 87\%).

${ }^{1} \mathrm{H}$ NMR $\left(400 \mathrm{MHz}, 298 \mathrm{~K}, \mathrm{DMSO}-d_{6}\right): \delta(\mathrm{ppm})=2.63\left(\mathrm{~s}, 3 \mathrm{H}, \mathrm{CH}_{3}\right), 7.53$ (br. s, $\left.1 \mathrm{H}, \mathrm{NH}\right)$, $7.61\left(\mathrm{t},{ }^{3} J_{\mathrm{H}-\mathrm{H}}=8.0 \mathrm{~Hz}, 1 \mathrm{H}, \mathrm{CH}_{\mathrm{Ar}}\right), 8.08-8.13\left(\mathrm{~m}, 2 \mathrm{H}, \mathrm{CH}_{\mathrm{Ar}}\right), 8.18$ (br. s, $\left.1 \mathrm{H}, \mathrm{NH}\right), 8.43(\mathrm{~m}$, $\left.1 \mathrm{H}, \mathrm{CH}_{\mathrm{Ar}}\right)$.

${ }^{13} \mathrm{C}-\left\{{ }^{1} \mathrm{H}\right\}$ NMR $\left.\left(100.62 \mathrm{MHz}, 298 \mathrm{~K}, \mathrm{DMSO}-d_{6}\right): \delta(\mathrm{ppm})=26.9 \mathrm{~s}, \mathrm{CH}_{3}\right), 127.2\left(\mathrm{~s}, \mathrm{CH}_{\mathrm{Ar}}\right)$, $128.8\left(\mathrm{~s}, \mathrm{CH}_{\mathrm{Ar}}\right), 130.7\left(\mathrm{~s}, \mathrm{CH}_{\mathrm{Ar}}\right), 132.0\left(\mathrm{~s}, \mathrm{CH}_{\mathrm{Ar}}\right), 134.7\left(\mathrm{~s}, \mathrm{C}_{\mathrm{Ar}}\right), 136.8\left(\mathrm{~s}, \mathrm{C}_{\mathrm{Ar}}\right), 167.1(\mathrm{~s}$, $\left.\mathrm{CONH}_{2}\right), 197.7\left(\mathrm{~s}, \mathrm{COCH}_{3}\right)$.

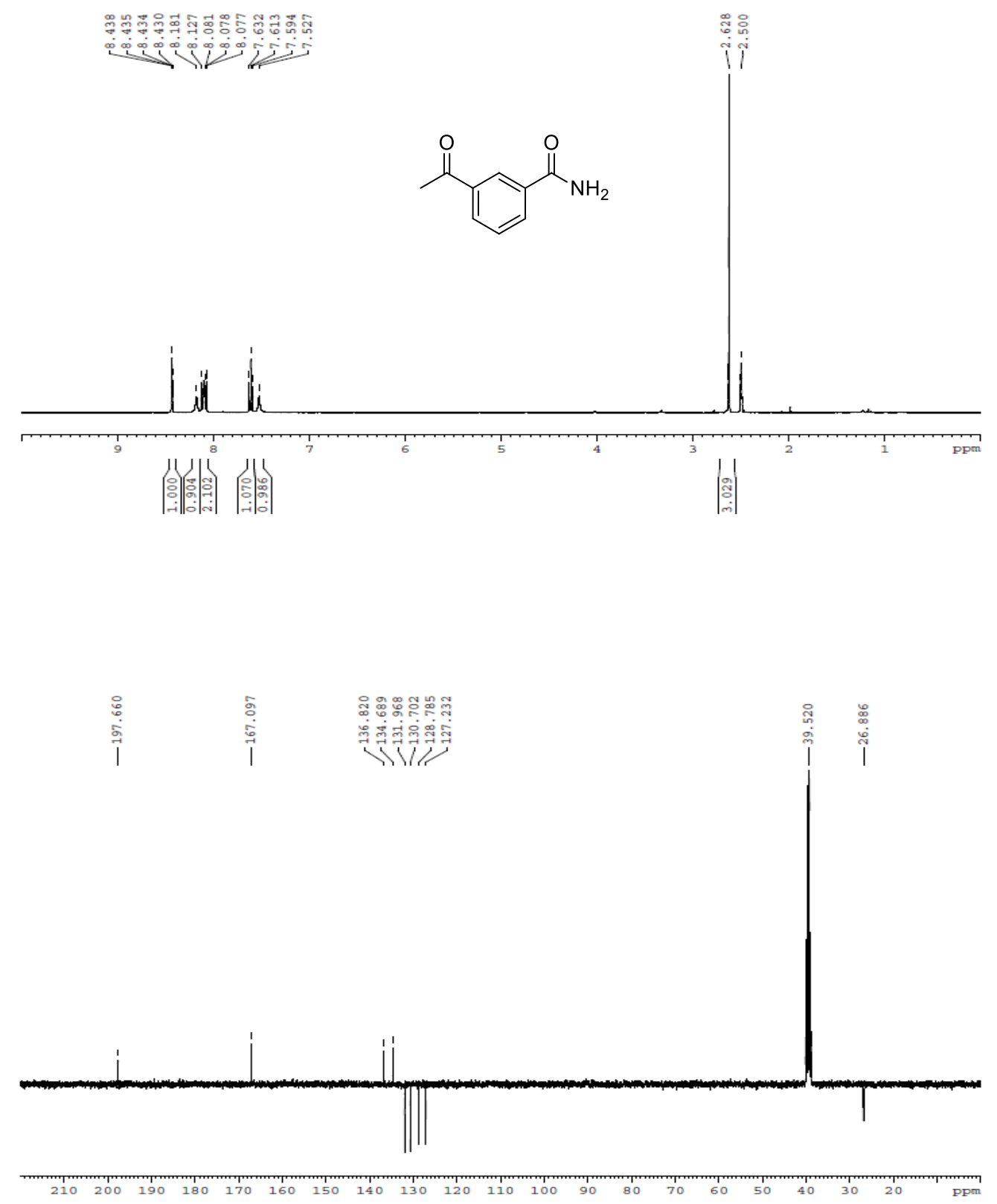




\section{$m$-Nitrobenzamide ${ }^{4}$}

The general procedure afforded the title compound (workup A) as a pale yellow solid (140 $\mathrm{mg}, 84 \%)$.

${ }^{1} \mathrm{H}$ NMR (400 MHz, 298K, DMSO- $\left.d_{6}\right): \delta(\mathrm{ppm})=7.72$ (br. s, $\left.1 \mathrm{H}, \mathrm{NH}\right), 7.77\left(\mathrm{t},{ }^{3} J_{\mathrm{H}-\mathrm{H}}=8.0\right.$ $\left.\mathrm{Hz}, 1 \mathrm{H}, \mathrm{CH}_{\mathrm{Ar}}\right), 8.29-8.32\left(\mathrm{~m}, 1 \mathrm{H}, \mathrm{CH}_{\mathrm{Ar}}\right), 8.34$ (br. s, $\left.1 \mathrm{H}, \mathrm{NH}\right), 8.36-8.40$ (m, $\left.1 \mathrm{H}, \mathrm{CH}_{\mathrm{Ar}}\right), 8.69$ $\left(\mathrm{t},{ }^{4} J_{\mathrm{H}-\mathrm{H}}=1.2 \mathrm{~Hz}, 1 \mathrm{H}, \mathrm{CH}_{\mathrm{Ar}}\right)$.

${ }^{13} \mathrm{C}-\left\{{ }^{1} \mathrm{H}\right\}$ NMR $\left(100.62 \mathrm{MHz}, 298 \mathrm{~K},\left(\mathrm{DMSO}-d_{6}\right): \delta(\mathrm{ppm})=122.2\left(\mathrm{~s}, \mathrm{CH}_{\mathrm{Ar}}\right), 125.9\left(\mathrm{~s}, \mathrm{CH}_{\mathrm{Ar}}\right)\right.$, $130.1\left(\mathrm{~s}, \mathrm{CH}_{\mathrm{Ar}}\right), 133.8\left(\mathrm{~s}, \mathrm{CH}_{\mathrm{Ar}}\right), 135.8\left(\mathrm{~s}, \mathrm{C}_{\mathrm{Ar}}\right), 147.8\left(\mathrm{~s}, \mathrm{C}_{\mathrm{Ar}}\right), 165.7\left(\mathrm{~s}, \mathrm{CONH}_{2}\right)$.
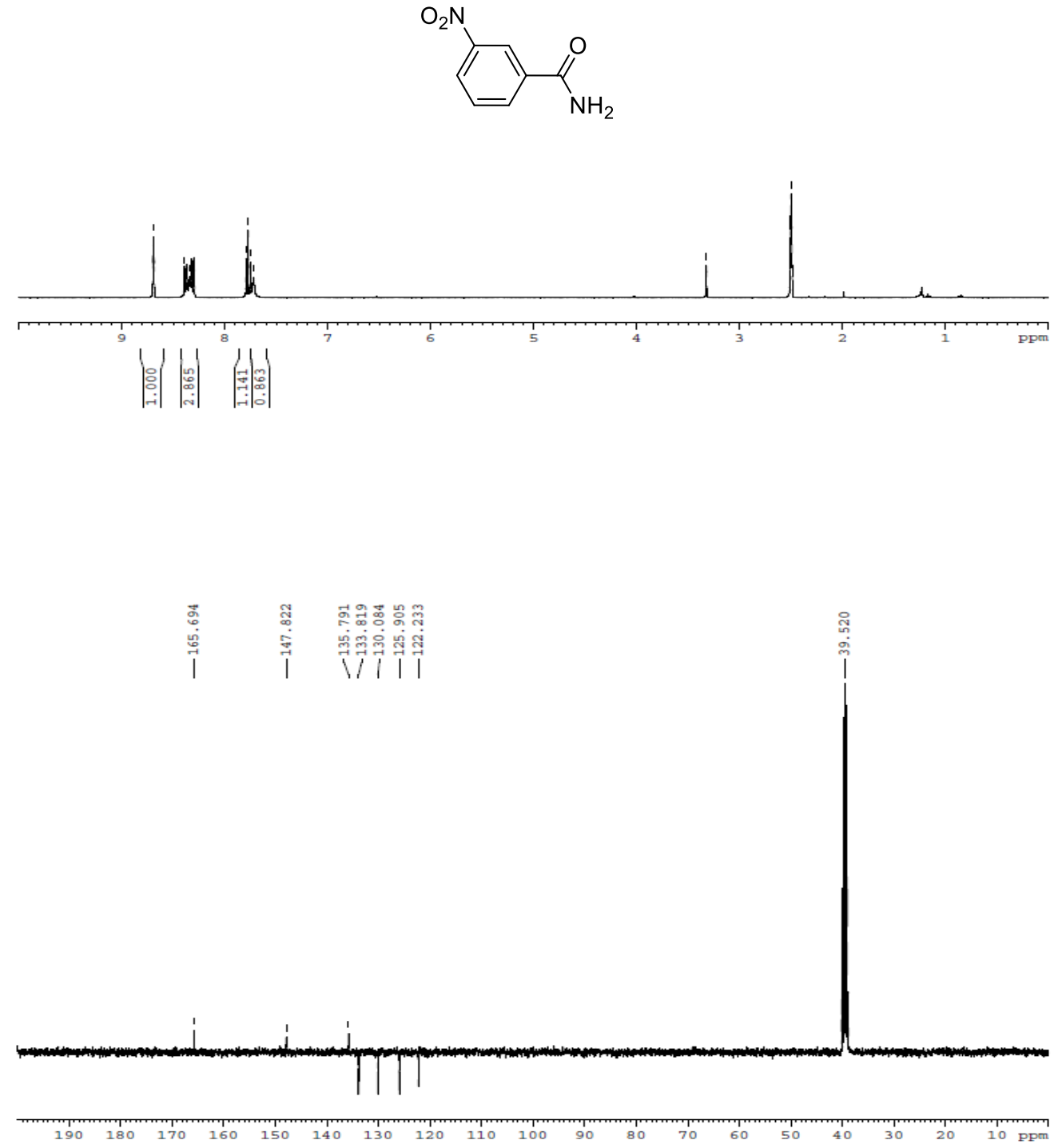


\section{$p$-(Trifluoromethyl)benzamide ${ }^{3}$}

The general procedure afforded the title compound (workup A) as a colourless solid (170 mg, $90 \%)$.

${ }^{1} \mathrm{H}$ NMR (400 MHz, 298K, DMSO- $\left.d_{6}\right): \delta(\mathrm{ppm})=7.63$ (br. s, $\left.1 \mathrm{H}, \mathrm{NH}\right), 7.83\left(\mathrm{~d},{ }^{3} J_{\mathrm{H}-\mathrm{H}}=8.0\right.$ $\left.\mathrm{Hz}, 2 \mathrm{H}, \mathrm{CH}_{\mathrm{Ar}}\right), 8.06\left(\mathrm{~d},{ }^{3} \mathrm{~J}_{\mathrm{H}-\mathrm{H}}=8.0 \mathrm{~Hz}, 2 \mathrm{H}, \mathrm{CH}_{\mathrm{Ar}}\right.$ ), 8.20 (br. s, $\left.1 \mathrm{H}, \mathrm{NH}\right)$.

${ }^{13} \mathrm{C}-\left\{{ }^{1} \mathrm{H}\right\}$ NMR $\left(100.62 \mathrm{MHz}, 298 \mathrm{~K}, \mathrm{DMSO}-d_{6}\right): \delta(\mathrm{ppm})=124.0\left(\mathrm{q},{ }^{1} J_{\mathrm{C}-\mathrm{F}}=271.4 \mathrm{~Hz}, \mathrm{CF}_{3}\right)$, $125.3\left(\mathrm{q},{ }^{3} J_{\mathrm{C}-\mathrm{F}}=3.6 \mathrm{~Hz}, \mathrm{CH}_{\mathrm{Ar}}\right), 128.4\left(\mathrm{~s}, \mathrm{CH}_{\mathrm{Ar}}\right), 131.2\left(\mathrm{~s},{ }^{2} J_{\mathrm{C}-\mathrm{F}}=31.9 \mathrm{~Hz}, \mathrm{C}_{\mathrm{Ar}}\right), 138.1\left(\mathrm{~s}, \mathrm{C}_{\mathrm{Ar}}\right)$, $166.8\left(\mathrm{~s}, \mathrm{CONH}_{2}\right)$.
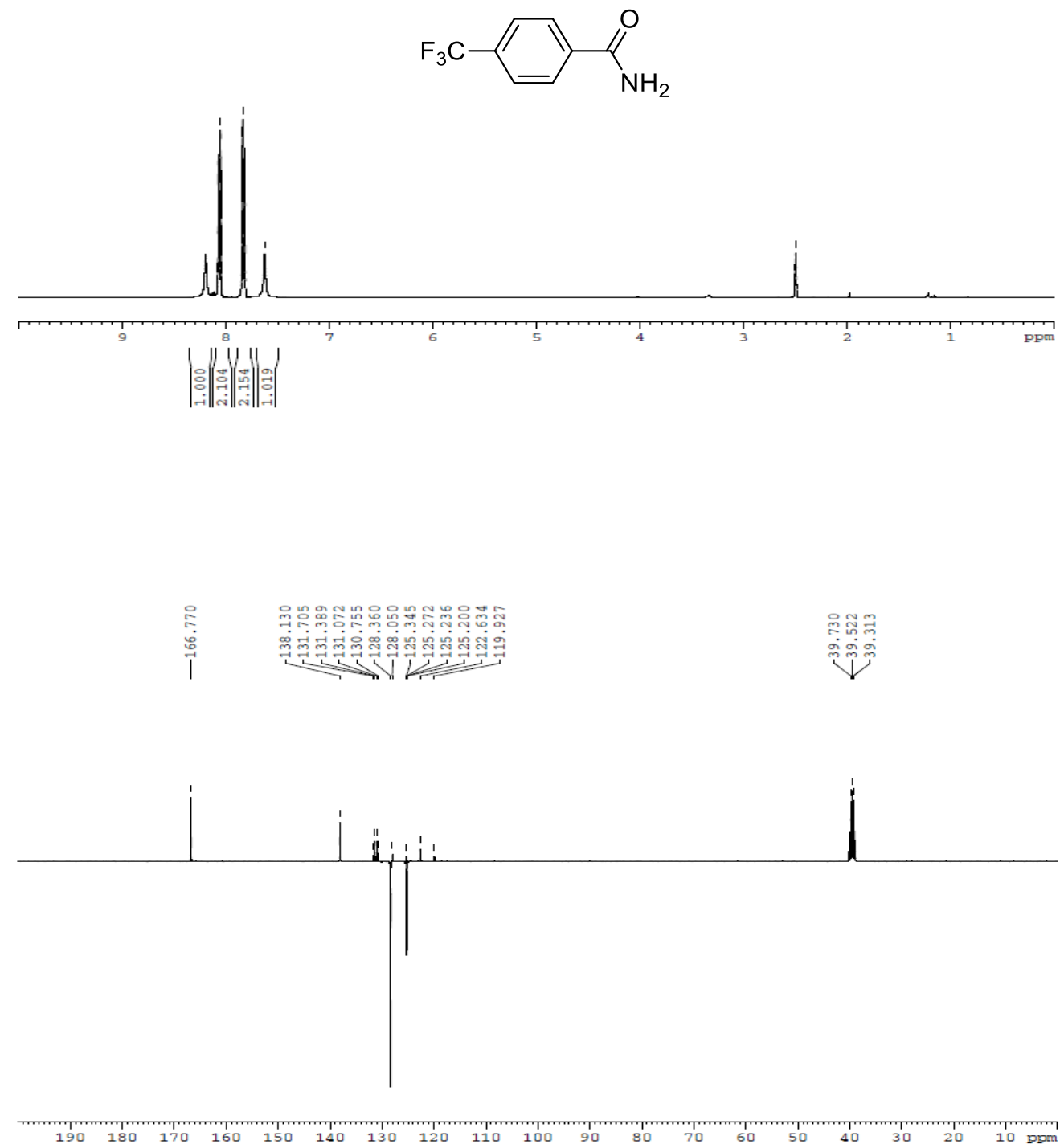


\section{$p$-Nitrobenzamide ${ }^{1}$}

The general procedure afforded the title compound (workup A) as a colourless solid (150 mg, $90 \%)$.

${ }^{1} \mathrm{H}$ NMR $\left(400 \mathrm{MHz}, 298 \mathrm{~K}, \mathrm{DMSO}-d_{6}\right): \delta(\mathrm{ppm})=7.73$ (br. s, $\left.1 \mathrm{H}, \mathrm{NH}\right), 8.08-8.11(\mathrm{~m}, 2 \mathrm{H}$, $\left.\mathrm{CH}_{\mathrm{Ar}}\right), 8.26-8.32\left(\mathrm{~m}, 3 \mathrm{H}, \mathrm{N} H+\mathrm{C} H_{\mathrm{Ar}}\right)$.

${ }^{13} \mathrm{C}-\left\{{ }^{1} \mathrm{H}\right\}$ NMR $\left(100.62 \mathrm{MHz}, 298 \mathrm{~K}, \mathrm{DMSO}-d_{6}\right): \delta(\mathrm{ppm})=123.5\left(\mathrm{~s}, \mathrm{CH}_{\mathrm{Ar}}\right), 128.9\left(\mathrm{~s}, \mathrm{CH}_{\mathrm{Ar}}\right)$, $140.0\left(\mathrm{~s}, \mathrm{C}_{\mathrm{Ar}}\right), 149.1\left(\mathrm{~s}, \mathrm{C}_{\mathrm{Ar}}\right), 166.2\left(\mathrm{~s}, \mathrm{CONH}_{2}\right)$.

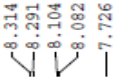
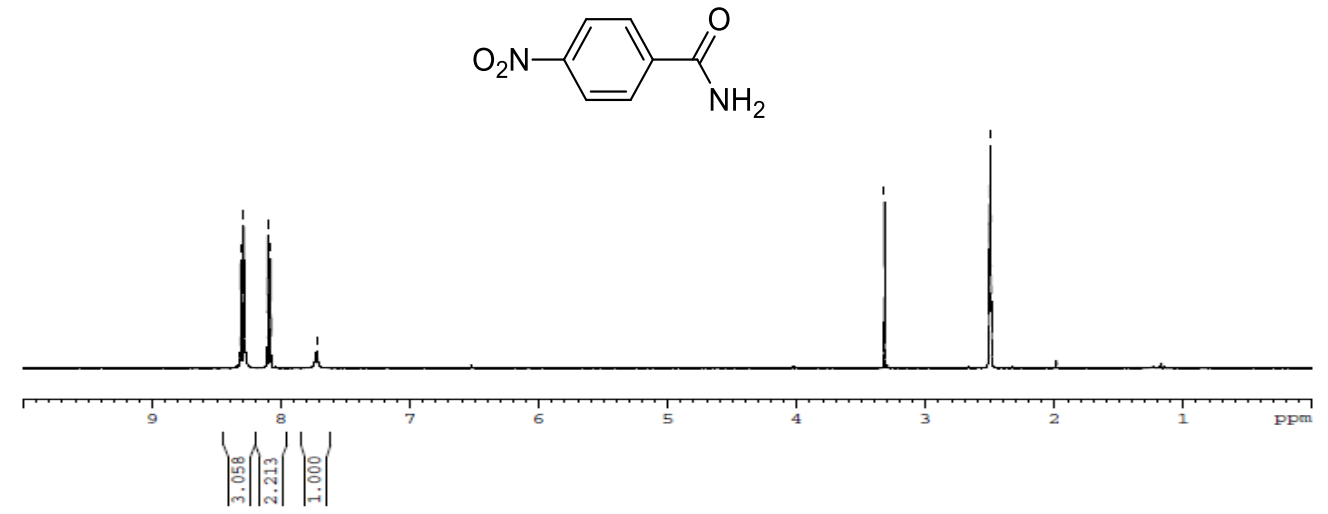

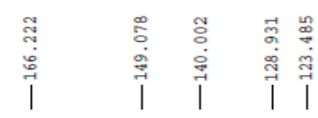

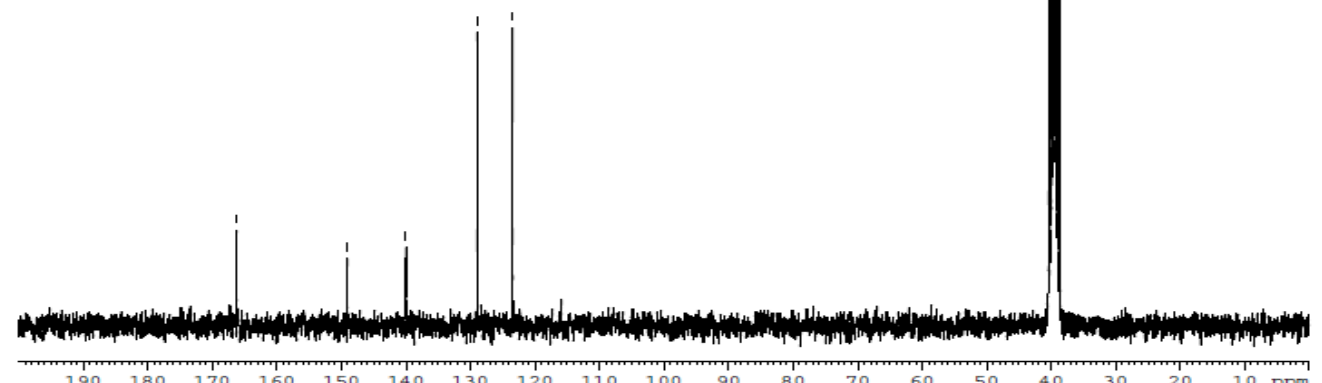




\section{p-Toluamide ${ }^{1}$}

The general procedure afforded the title compound (workup A) as a colourless solid (114 mg, $84 \%$ ).

${ }^{1} \mathrm{H}$ NMR $\left(400 \mathrm{MHz}, 298 \mathrm{~K}, \mathrm{DMSO}-d_{6}\right): \delta(\mathrm{ppm})=2.34\left(\mathrm{~s}, 3 \mathrm{H}, \mathrm{CH}_{3}\right), 7.22-7.29(\mathrm{~m}, 3 \mathrm{H}, \mathrm{NH}+$ $\left.\mathrm{CH}_{\mathrm{Ar}}\right), 7.77\left(\mathrm{~d},{ }^{3} \mathrm{~J}_{\mathrm{H}-\mathrm{H}}=8.0 \mathrm{~Hz}, 2 \mathrm{H}, \mathrm{CH}_{\mathrm{Ar}}\right), 7.88$ (br. s, $1 \mathrm{H}, \mathrm{NH}$ ).

${ }^{13} \mathrm{C}-\left\{{ }^{1} \mathrm{H}\right\}$ NMR $\left(100.62 \mathrm{MHz}, 298 \mathrm{~K}, \mathrm{DMSO}-d_{6}\right): \delta(\mathrm{ppm})=21.0\left(\mathrm{~s}, \mathrm{CH}_{3}\right), 127.5\left(\mathrm{~s}, \mathrm{CH}_{\mathrm{Ar}}\right)$, $128.7\left(\mathrm{~s}, \mathrm{CH}_{\mathrm{Ar}}\right), 131.5\left(\mathrm{~s}, \mathrm{C}_{\mathrm{Ar}}\right), 141.1\left(\mathrm{~s}, \mathrm{C}_{\mathrm{Ar}}\right), 167.8\left(\mathrm{~s}, \mathrm{CONH}_{2}\right)$.
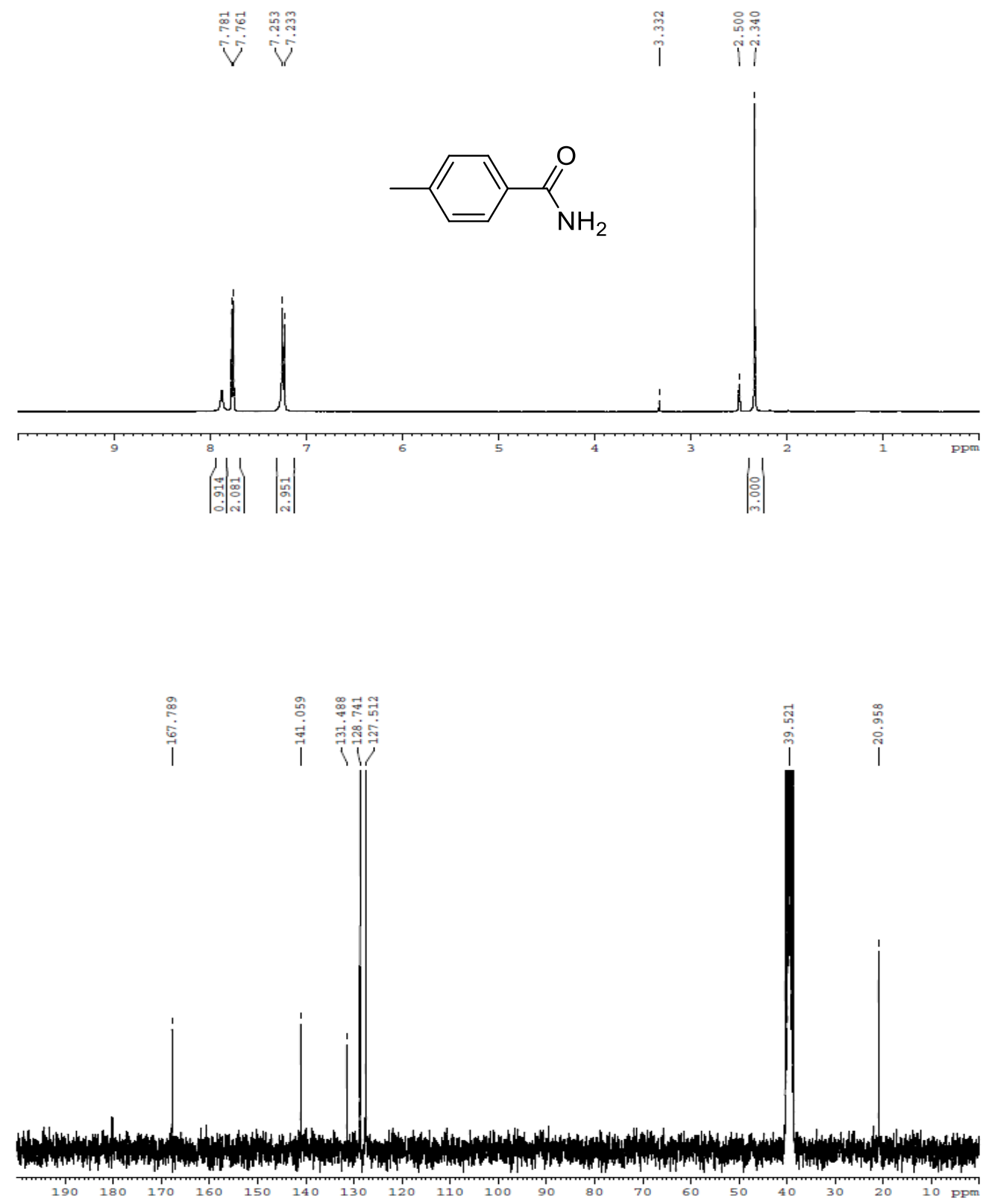


\section{p-Acetylbenzamide ${ }^{3}$}

The general procedure afforded the title compound (workup A) as a colourless solid (137 mg, $84 \%)$.

${ }^{1} \mathrm{H}$ NMR $\left(400 \mathrm{MHz}, 298 \mathrm{~K}, \mathrm{DMSO}-d_{6}\right): \delta(\mathrm{ppm})=2.61\left(\mathrm{~s}, 3 \mathrm{H}, \mathrm{CH}_{3}\right), 7.57$ (br. s, $\left.1 \mathrm{H}, \mathrm{NH}\right)$, 7.97-8.02 (m, $\left.4 \mathrm{H}, \mathrm{CH}_{\mathrm{Ar}}\right), 8.15$ (br. s, $\left.1 \mathrm{H}, \mathrm{NH}\right)$.

${ }^{13} \mathrm{C}-\left\{{ }^{1} \mathrm{H}\right\}$ NMR $\left(100.62 \mathrm{MHz}, 298 \mathrm{~K}, \mathrm{DMSO}-d_{6}\right): \delta(\mathrm{ppm})=27.0\left(\mathrm{~s}, \mathrm{CH}_{3}\right), 127.8\left(\mathrm{~s}, \mathrm{CH}_{\mathrm{Ar}}\right)$, $128.1\left(\mathrm{~s}, \mathrm{CH}_{\mathrm{Ar}}\right), 138.1\left(\mathrm{~s}, \mathrm{C}_{\mathrm{Ar}}\right), 138.7\left(\mathrm{~s}, \mathrm{C}_{\mathrm{Ar}}\right), 167.1\left(\mathrm{~s}, \mathrm{CONH}_{2}\right), 197.7\left(\mathrm{~s}, \mathrm{COCH}_{3}\right)$.

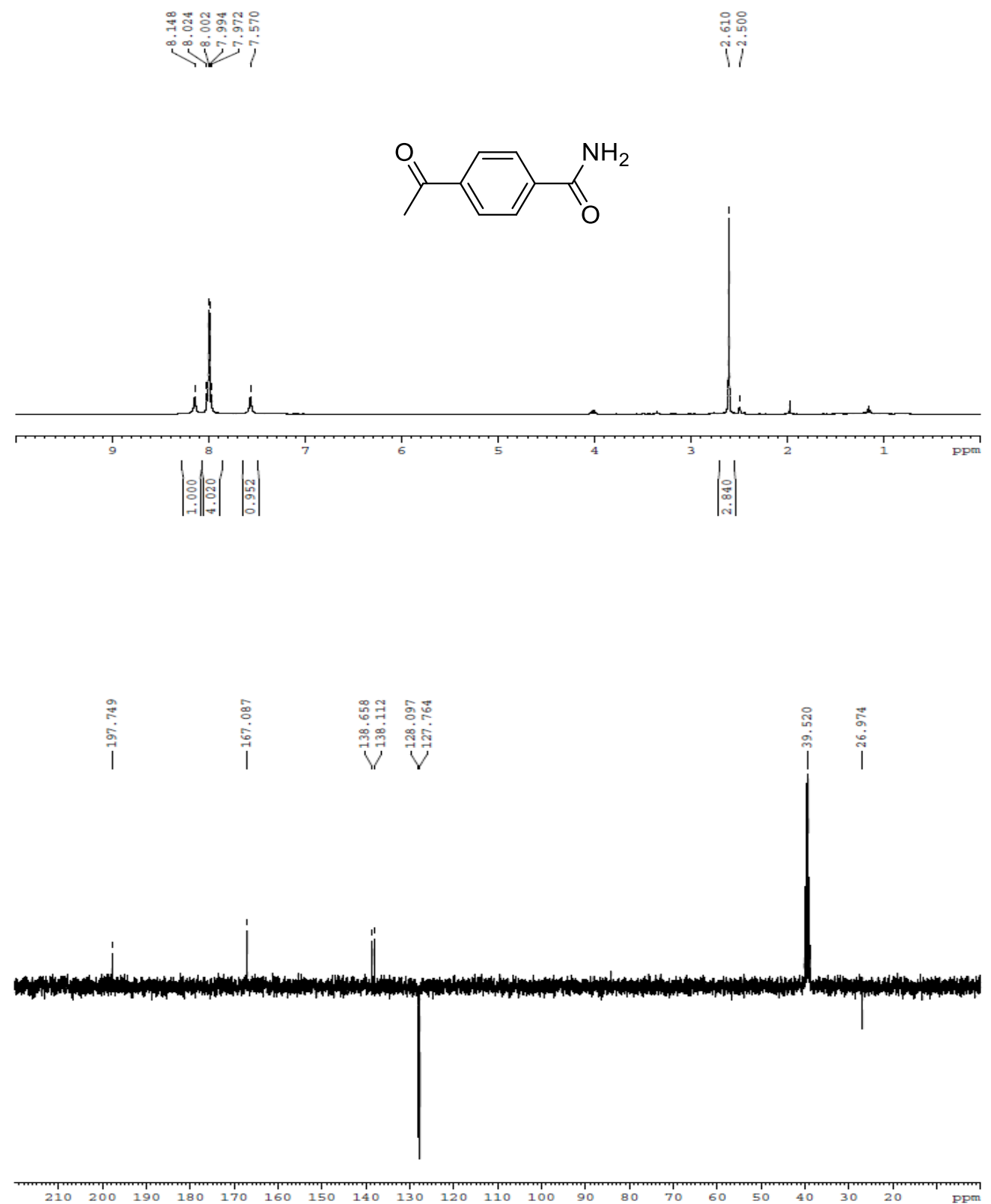




\section{p-Fluorobenzamide ${ }^{5}$}

The general procedure afforded the title compound (workup A) as a colourless solid (134 mg, $96 \%)$.

${ }^{1} \mathrm{H}$ NMR (400 MHz, 298K, DMSO-d $\left.d_{6}\right): \delta(\mathrm{ppm})=7.25-7.30\left(\mathrm{~m}, 2 \mathrm{H}, \mathrm{CH}_{\mathrm{Ar}}\right), 7.39$ (br. s, $1 \mathrm{H}$, $\mathrm{NH})$, 7.92-7.96 (m, 2H, $\left.\mathrm{CH}_{\mathrm{Ar}}\right), 7.99$ (br. s, 1H, NH).

${ }^{13} \mathrm{C}-\left\{{ }^{1} \mathrm{H}\right\}$ NMR $\left(100.62 \mathrm{MHz}, 298 \mathrm{~K}, \mathrm{DMSO}-d_{6}\right): \delta(\mathrm{ppm})=115.1\left(\mathrm{~d},{ }^{2} J_{\mathrm{C}-\mathrm{F}}=20.7 \mathrm{~Hz}, \mathrm{CH}_{\mathrm{Ar}}\right)$, $130.1\left(\mathrm{~d},{ }^{3} J_{\mathrm{C}-\mathrm{F}}=8.1 \mathrm{~Hz}, \mathrm{CH}_{\mathrm{Ar}}\right), 130.7\left(\mathrm{~d},{ }^{4} J_{\mathrm{C}-\mathrm{F}}=2.7 \mathrm{~Hz}, \mathrm{C}_{\mathrm{Ar}}\right), 163.9\left(\mathrm{~d},{ }^{1} J_{\mathrm{C}-\mathrm{F}}=248.2 \mathrm{~Hz}, \mathrm{C}_{\mathrm{Ar}}\right)$, $166.8\left(\mathrm{~s}, \mathrm{CONH}_{2}\right)$.
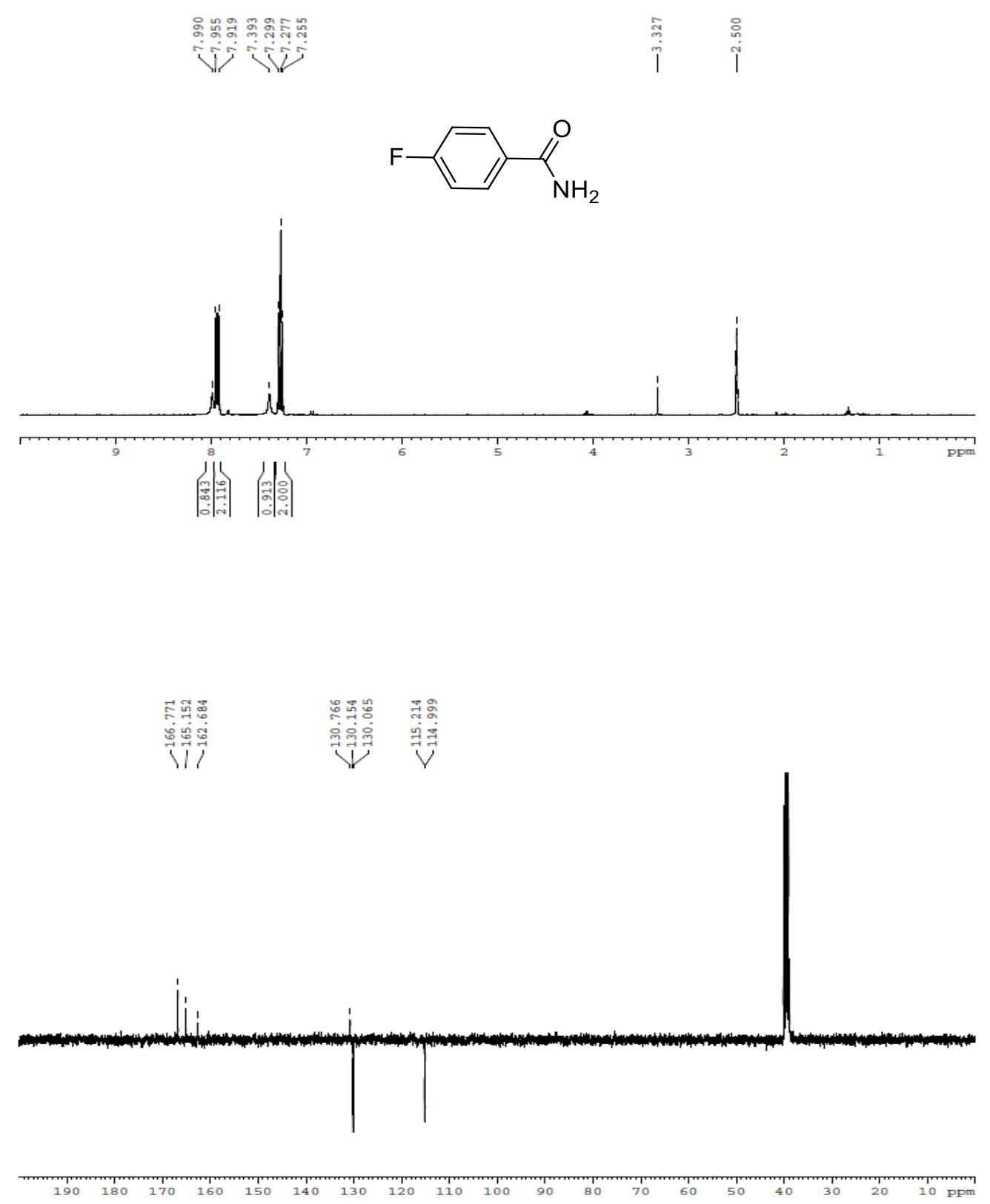


\section{p-Methoxybenzamide ${ }^{1}$}

The general procedure afforded the title compound (workup A) as a colourless solid (130 mg, $86 \%)$.

${ }^{1} \mathrm{H}$ NMR (400 MHz, 298K, DMSO- $\left.d_{6}\right): \delta(\mathrm{ppm})=3.80\left(\mathrm{~s}, 3 \mathrm{H}, \mathrm{OCH}_{3}\right), 6.96-6.98(\mathrm{~m}, 2 \mathrm{H}$, $\mathrm{CH}_{\mathrm{Ar}}$ ), 7.17 (br. s, $\left.1 \mathrm{H}, \mathrm{NH}\right), 7.82-7.86\left(\mathrm{~m}, 3 \mathrm{H}, \mathrm{N} H+\mathrm{CH}_{\mathrm{Ar}}\right)$.

${ }^{13} \mathrm{C}-\left\{{ }^{1} \mathrm{H}\right\}$ NMR $\left(100.62 \mathrm{MHz}, 298 \mathrm{~K}, \mathrm{DMSO}-d_{6}\right): \delta(\mathrm{ppm})=55.3\left(\mathrm{~s}, \mathrm{OCH}_{3}\right), 113.4\left(\mathrm{~s}, \mathrm{CH}_{\mathrm{Ar}}\right)$, $126.5\left(\mathrm{~s}, \mathrm{C}_{\mathrm{Ar}}\right), 129.4\left(\mathrm{~s}, \mathrm{CH}_{\mathrm{Ar}}\right), 161.6\left(\mathrm{~s}, \mathrm{C}_{\mathrm{Ar}}\right), 167.4\left(\mathrm{~s}, \mathrm{CONH}_{2}\right)$.

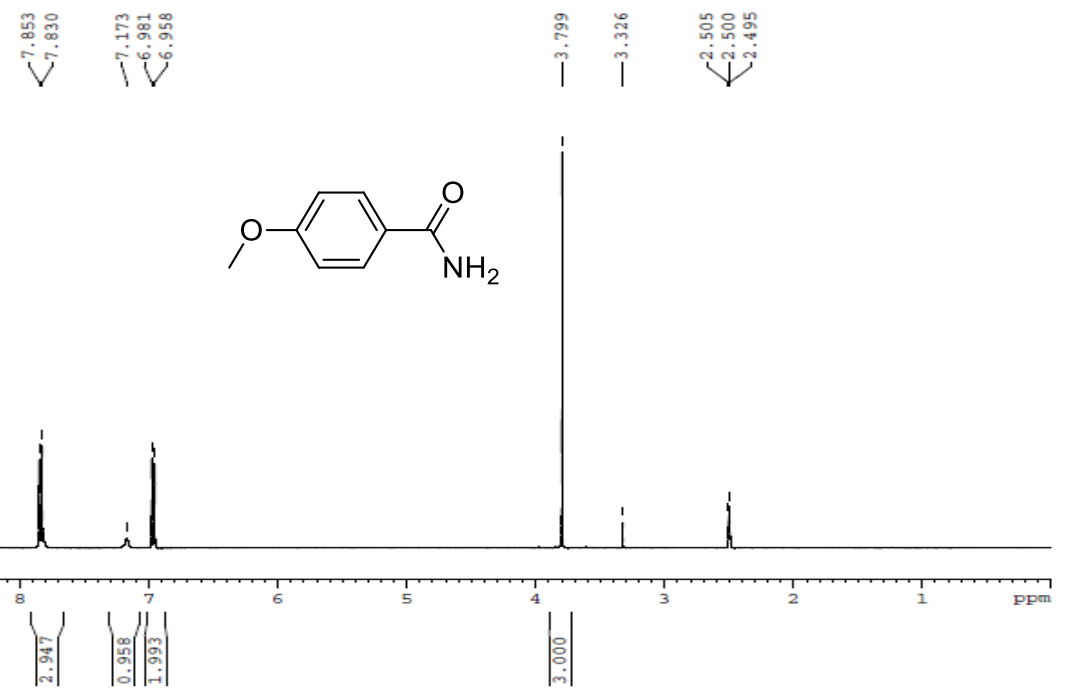

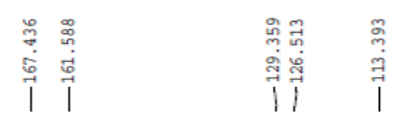

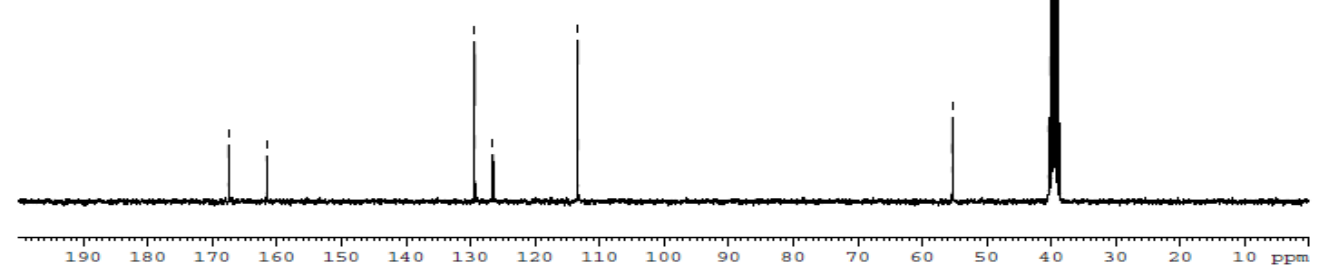




\section{p-Chlorobenzamide ${ }^{1}$}

The general procedure afforded the title compound (workup A) as a colourless solid (140 mg, $90 \%)$.

${ }^{1} \mathrm{H}$ NMR $\left(400 \mathrm{MHz}, 298 \mathrm{~K}, \mathrm{DMSO}-d_{6}\right): \delta(\mathrm{ppm})=7.46$ (br. s, $\left.1 \mathrm{H}, N H\right), 7.51-7.54(\mathrm{~m}, 2 \mathrm{H}$, $\left.\mathrm{CH}_{A r}\right)$, 7.87-7.89 (m, 2H, $\left.\mathrm{CH}_{A r}\right), 8.04$ (br. s, 2H, NH).

${ }^{13} \mathrm{C}-\left\{{ }^{1} \mathrm{H}\right\}$ NMR $\left(100.62 \mathrm{MHz}, 298 \mathrm{~K}, \mathrm{DMSO}-d_{6}\right): \delta(\mathrm{ppm})=128.3\left(\mathrm{~s}, \mathrm{CH}_{\mathrm{Ar}}\right), 129.4\left(\mathrm{~s}, \mathrm{CH}_{\mathrm{Ar}}\right)$, $133.0\left(\mathrm{~s}, \mathrm{C}_{\mathrm{Ar}}\right), 136.1\left(\mathrm{~s}, \mathrm{CH}_{\mathrm{Ar}}\right), 166.8\left(\mathrm{~s}, \mathrm{CONH}_{2}\right)$.
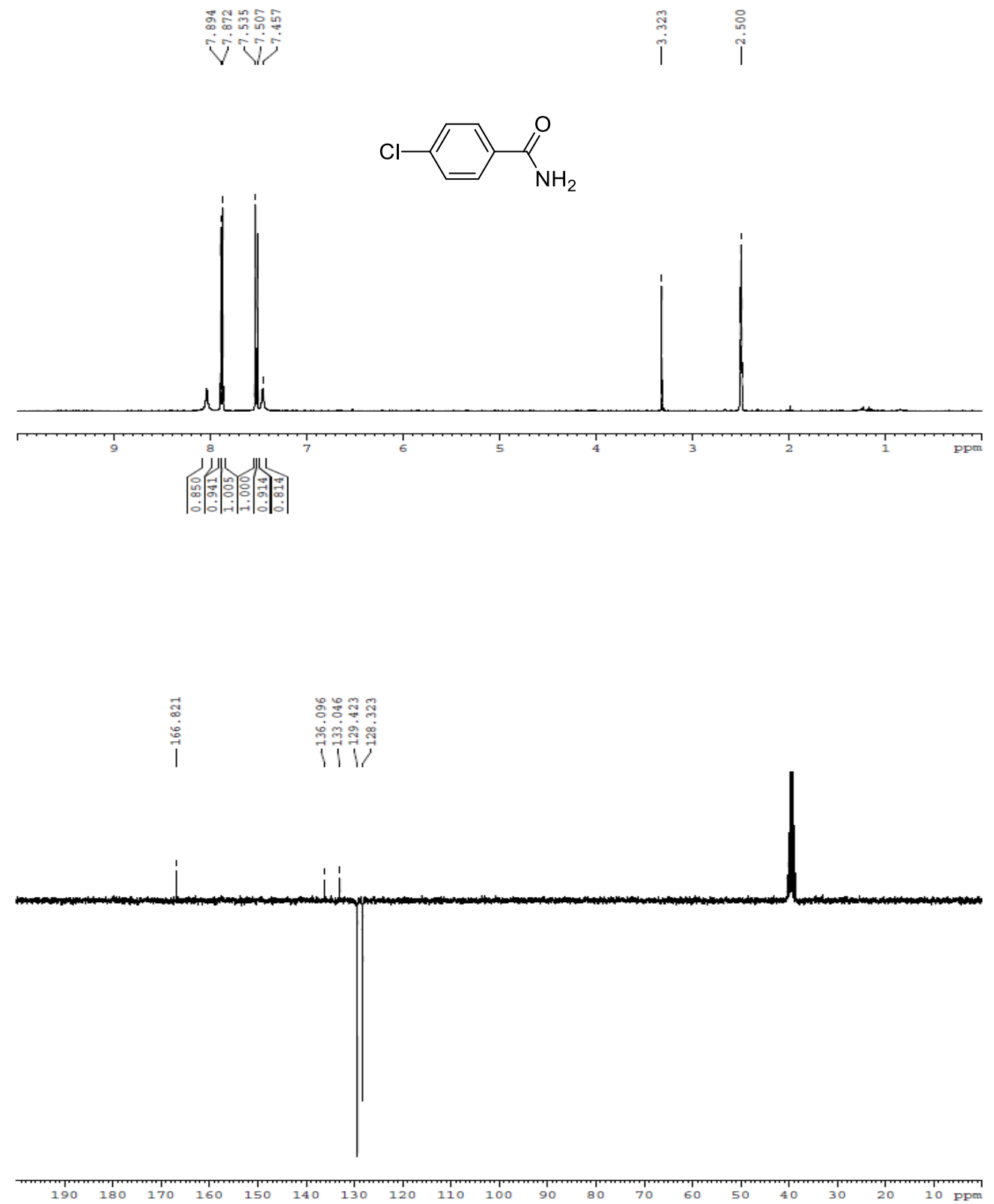


\section{Isophthalamide ${ }^{1}$}

The general procedure afforded the title compound (workup B) as a colourless solid (99 mg, $60 \%)$.

${ }^{1} \mathrm{H}$ NMR $\left(400 \mathrm{MHz}, 298 \mathrm{~K}\right.$, DMSO- $\left.d_{6}\right): \delta(\mathrm{ppm})=7.46$ (br. s, $\left.2 \mathrm{H}, \mathrm{N} H_{2}\right), 7.53\left(\mathrm{t},{ }^{3} J_{\mathrm{H}-\mathrm{H}}=8.0\right.$ $\left.\mathrm{Hz}, 1 \mathrm{H}, \mathrm{C} H_{\mathrm{Ar}}\right), 7.99\left(\mathrm{dd},{ }^{3} J_{\mathrm{H}-\mathrm{H}}=8.0 \mathrm{~Hz},{ }^{4} J_{\mathrm{H}-\mathrm{H}}=1.2 \mathrm{~Hz}, 2 \mathrm{H}, \mathrm{C} H_{\mathrm{Ar}}\right), 8.03$ (br. s, $\left.2 \mathrm{H}, \mathrm{N} H\right), 8.36$ $\left(\mathrm{t},{ }^{4} J_{\mathrm{H}-\mathrm{H}}=1.2 \mathrm{~Hz}, 1 \mathrm{H}, \mathrm{C} H_{\mathrm{Ar}}\right)$.

${ }^{13} \mathrm{C}-\left\{{ }^{1} \mathrm{H}\right\}$ NMR $\left(100.62 \mathrm{MHz}, 298 \mathrm{~K}, \mathrm{DMSO}-d_{6}\right): \delta(\mathrm{ppm})=126.8\left(\mathrm{~s}, \mathrm{CH}_{\mathrm{Ar}}\right), 128.3\left(\mathrm{~s}, \mathrm{CH}_{\mathrm{Ar}}\right)$, $130.1\left(\mathrm{~s}, C \mathrm{H}_{\mathrm{Ar}}\right), 134.5\left(\mathrm{~s}, C_{\mathrm{Ar}}\right), 167.6\left(\mathrm{~s}, C \mathrm{ONH}_{2}\right)$.
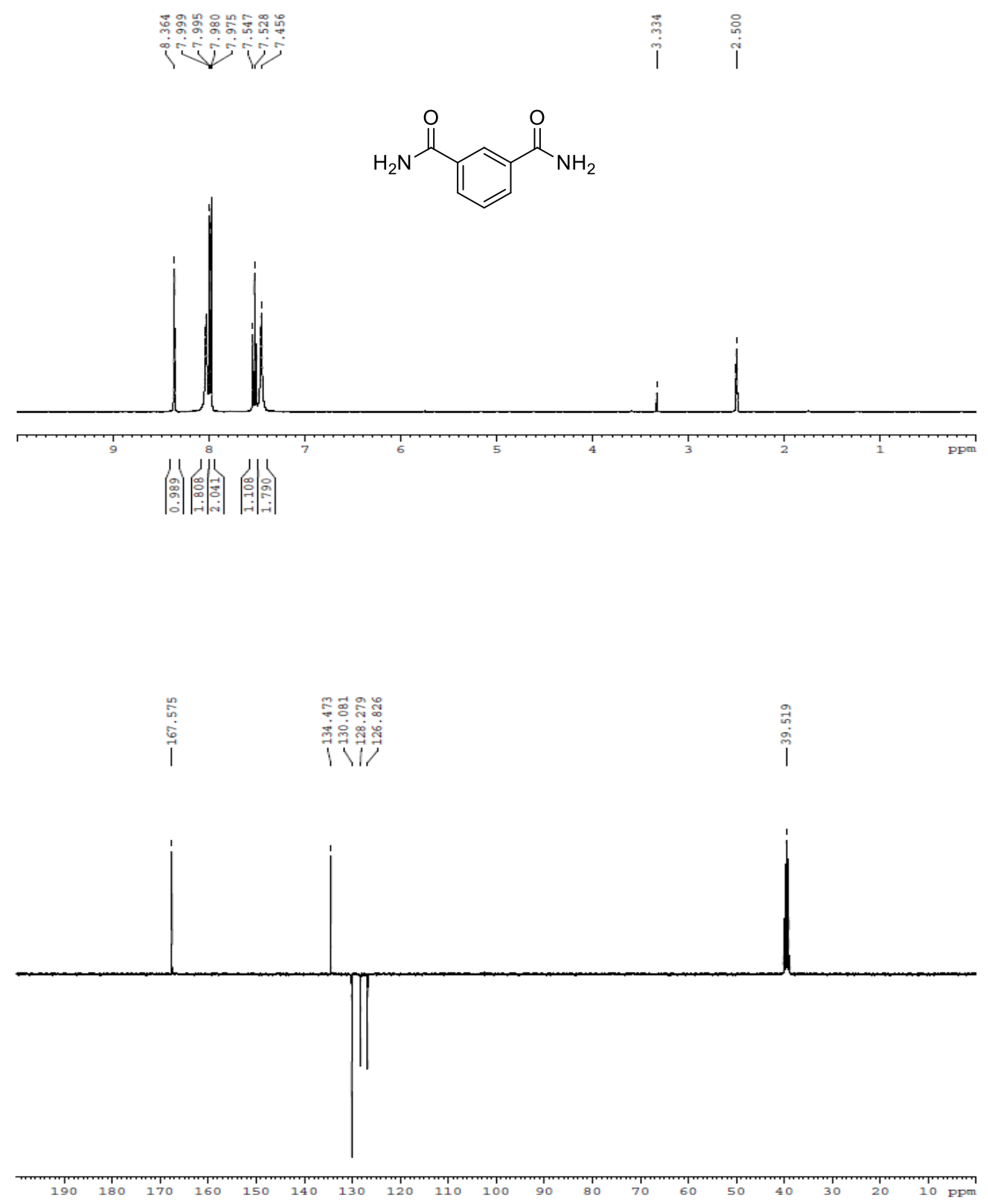


\section{Terephthalamide ${ }^{1}$}

The general procedure afforded the title compound (workup B) as a colourless solid (135 mg, $82 \%)$.

${ }^{1} \mathrm{H}$ NMR $\left(400 \mathrm{MHz}, 298 \mathrm{~K}, \mathrm{DMSO}-d_{6}\right): \delta(\mathrm{ppm})=7.49$ (br. s, 2H, NH), $7.92\left(\mathrm{~s}, 4 \mathrm{H}, \mathrm{CH}_{\mathrm{Ar}}\right)$, 8.07 (br. s, 2H, NH).

${ }^{13} \mathrm{C}-\left\{{ }^{1} \mathrm{H}\right\}$ NMR $\left(100.62 \mathrm{MHz}, 298 \mathrm{~K}, \mathrm{DMSO}-d_{6}\right): \delta(\mathrm{ppm})=127.4\left(\mathrm{~s}, \mathrm{CH}_{\mathrm{Ar}}\right), 136.6\left(\mathrm{~s}, \mathrm{C}_{\mathrm{Ar}}\right)$, $167.3\left(\mathrm{~s}, \mathrm{CONH}_{2}\right)$.

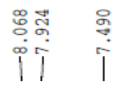

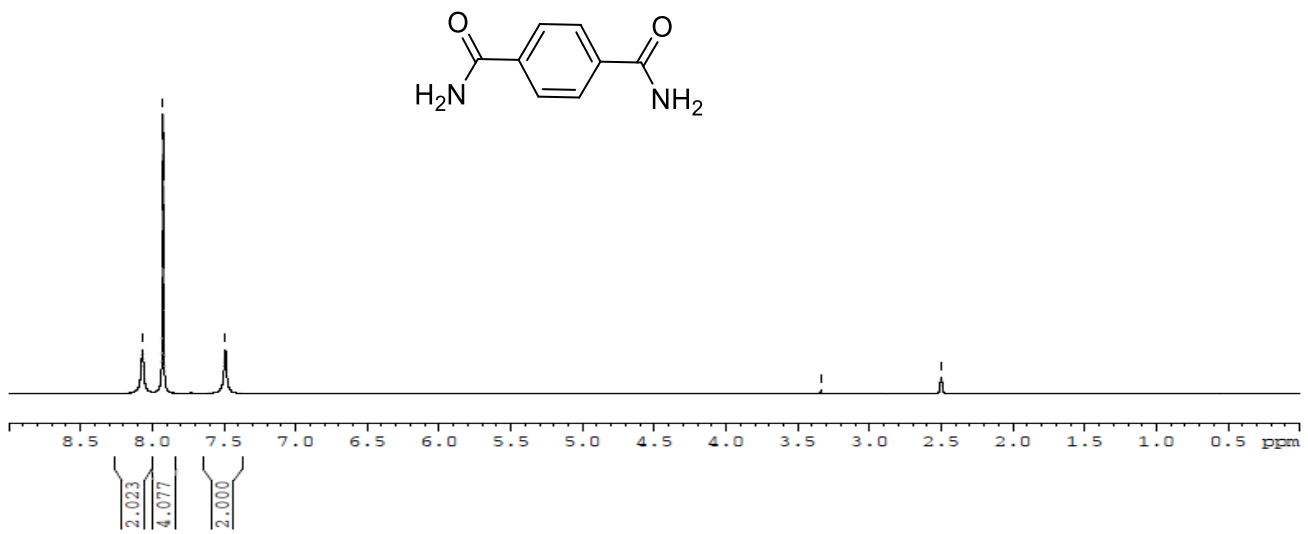

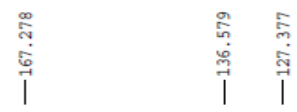

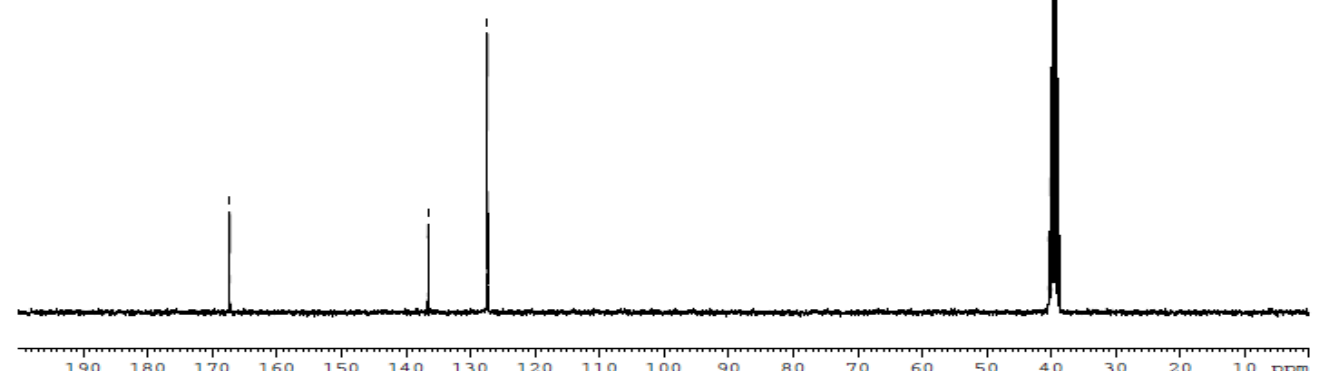




\section{2-Furamide ${ }^{1}$}

The general procedure afforded the title compound (workup A) as a colourless solid (98 mg, $88 \%)$.

${ }^{1} \mathrm{H}$ NMR $\left(400 \mathrm{MHz}, 298 \mathrm{~K}, \mathrm{DMSO}-d_{6}\right): \delta(\mathrm{ppm})=6.59-6.60\left(\mathrm{~m}, 1 \mathrm{H}, \mathrm{CH}_{\mathrm{Ar}}\right), 7.09\left(\mathrm{~d},{ }^{3} J_{\mathrm{H}-\mathrm{H}}=\right.$ $\left.3.3 \mathrm{~Hz}, 1 \mathrm{H}, \mathrm{CH} \mathrm{Ar}_{\mathrm{Ar}}\right), 7.36$ (br. s, $\left.1 \mathrm{H}, \mathrm{NH}\right), 7.75$ (br. s, $\left.1 \mathrm{H}, \mathrm{NH}\right), 7.80\left(\mathrm{~m}, 1 \mathrm{H}, \mathrm{CH}_{\mathrm{Ar}}\right)$.

${ }^{13} \mathrm{C}-\left\{{ }^{1} \mathrm{H}\right\}$ NMR $\left(100.62 \mathrm{MHz}, 298 \mathrm{~K}, \mathrm{DMSO}-d_{6}\right): \delta(\mathrm{ppm})=111.8\left(\mathrm{~s}, \mathrm{CH}_{\mathrm{Ar}}\right), 113.6\left(\mathrm{~s}, \mathrm{CH}_{\mathrm{Ar}}\right)$, $145.0\left(\mathrm{~s}, \mathrm{CH}_{\mathrm{Ar}}\right), 148.1\left(\mathrm{~s}, \mathrm{C}_{\mathrm{Ar}}\right), 159.4\left(\mathrm{~s}, \mathrm{CONH}_{2}\right)$.

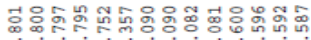

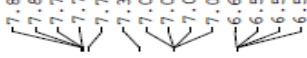
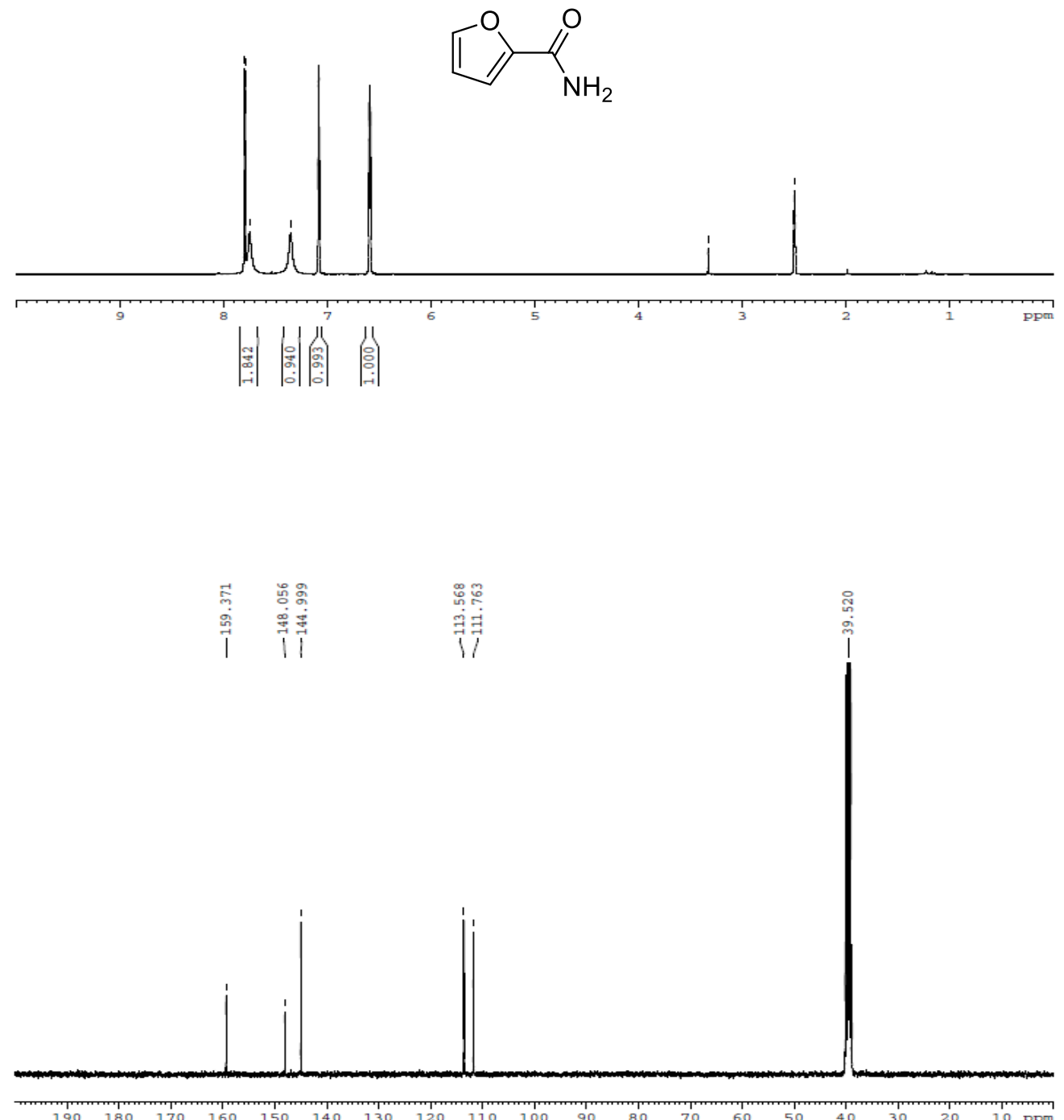


\section{Isonicotinamide ${ }^{1}$}

The general procedure afforded the title compound (workup A) as a colourless solid (88 $\mathrm{mg}$, $72 \%)$.

${ }^{1} \mathrm{H}$ NMR $\left(400 \mathrm{MHz}, 298 \mathrm{~K}, \mathrm{DMSO}-d_{6}\right): \delta(\mathrm{ppm})=7.72$ (br. s, $\left.1 \mathrm{H}, \mathrm{NH}\right), 7.75-7.77(\mathrm{~m}, 2 \mathrm{H}$, $\left.\mathrm{CH}_{\mathrm{Ar}}\right), 8.24$ (br. s, $\left.1 \mathrm{H}, \mathrm{NH}\right), 8.70-8.72\left(\mathrm{~m}, 2 \mathrm{H}, \mathrm{CH}_{\mathrm{Ar}}\right)$.

${ }^{13} \mathrm{C}-\left\{{ }^{1} \mathrm{H}\right\}$ NMR $\left(100.62 \mathrm{MHz}, 298 \mathrm{~K}, \mathrm{DMSO}-d_{6}\right): \delta(\mathrm{ppm})=121.4\left(\mathrm{~s}, \mathrm{CH}_{\mathrm{Ar}}\right), 141.3\left(\mathrm{~s}, \mathrm{C}_{\mathrm{Ar}}\right)$, $150.2\left(\mathrm{~s}, \mathrm{CH}_{\mathrm{Ar}}\right), 166.3\left(\mathrm{~s}, \mathrm{CONH}_{2}\right)$.
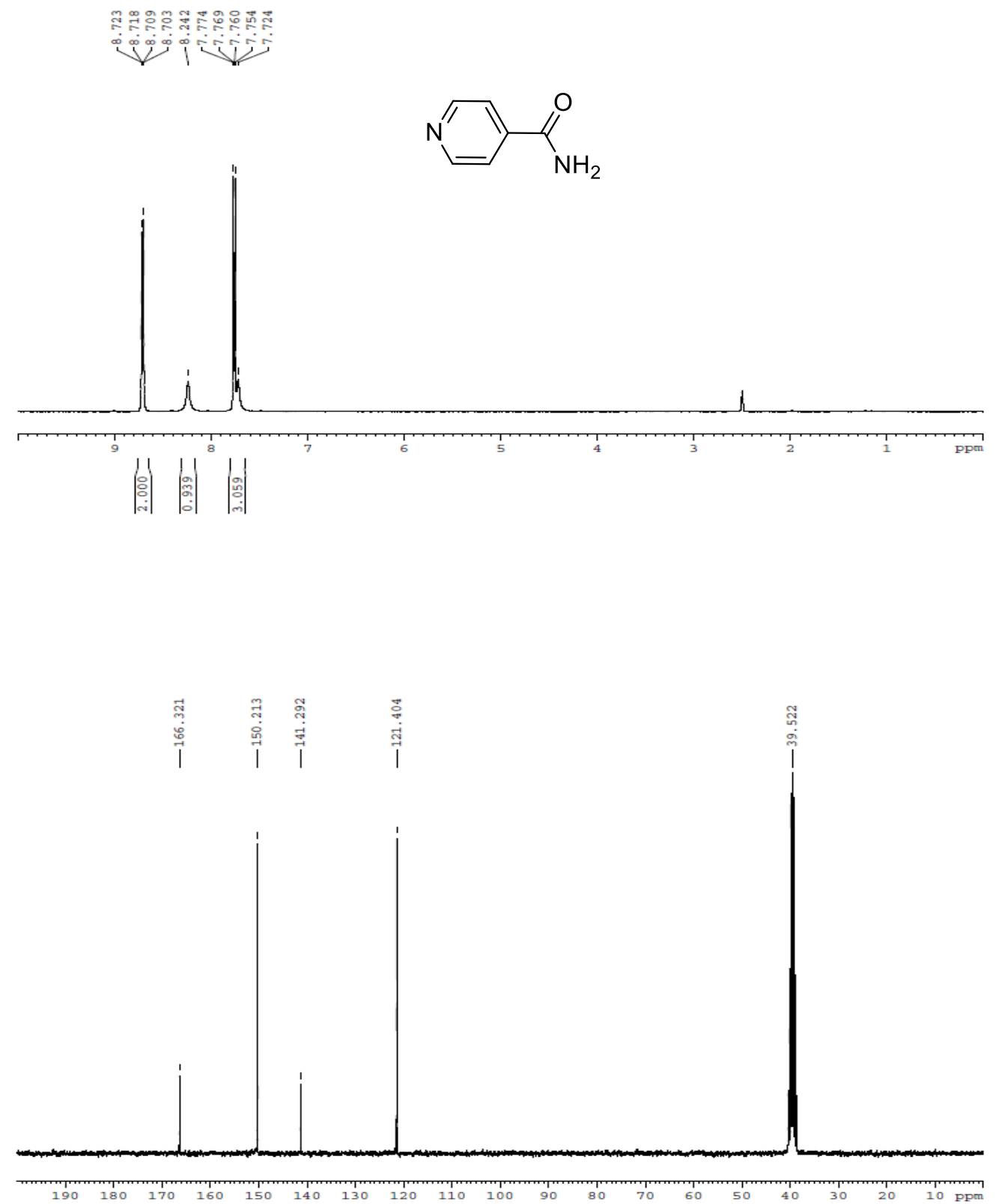


\section{H-Pyrrole-2-carboxamide ${ }^{1}$}

The general procedure afforded the title compound (workup A) as a colourless solid (96 mg, $87 \%)$.

${ }^{1} \mathrm{H}$ NMR $\left(400 \mathrm{MHz}, 298 \mathrm{~K}, \mathrm{DMSO}-d_{6}\right): \delta(\mathrm{ppm})=6.06\left(\mathrm{~m}, 1 \mathrm{H}, \mathrm{CH}_{\mathrm{Ar}}\right), 6.76\left(\mathrm{~m}, 1 \mathrm{H}, \mathrm{CH}_{\mathrm{Ar}}\right)$, $6.83\left(\mathrm{~m}, 1 \mathrm{H}, \mathrm{CH}_{\mathrm{Ar}}\right.$ ), 6.92 (br. s, 1H, NH), 7.47 (br. s, 1H, NH), 11.39 (s, 1H, $\mathrm{NH}_{\mathrm{Ar}}$ ).

${ }^{13} \mathrm{C}-\left\{{ }^{1} \mathrm{H}\right\}$ NMR $\left(100.62 \mathrm{MHz}, 298 \mathrm{~K}, \mathrm{DMSO}-d_{6}\right): \delta(\mathrm{ppm})=108.6\left(\mathrm{~s}, \mathrm{CH}_{\mathrm{Ar}}\right), 110.7\left(\mathrm{~s}, \mathrm{CH}_{\mathrm{Ar}}\right)$, $121.4\left(\mathrm{~s}, \mathrm{CH}_{\mathrm{Ar}}\right), 126.3\left(\mathrm{~s}, \mathrm{C}_{\mathrm{Ar}}\right), 162.3\left(\mathrm{~s}, \mathrm{CONH}_{2}\right)$.
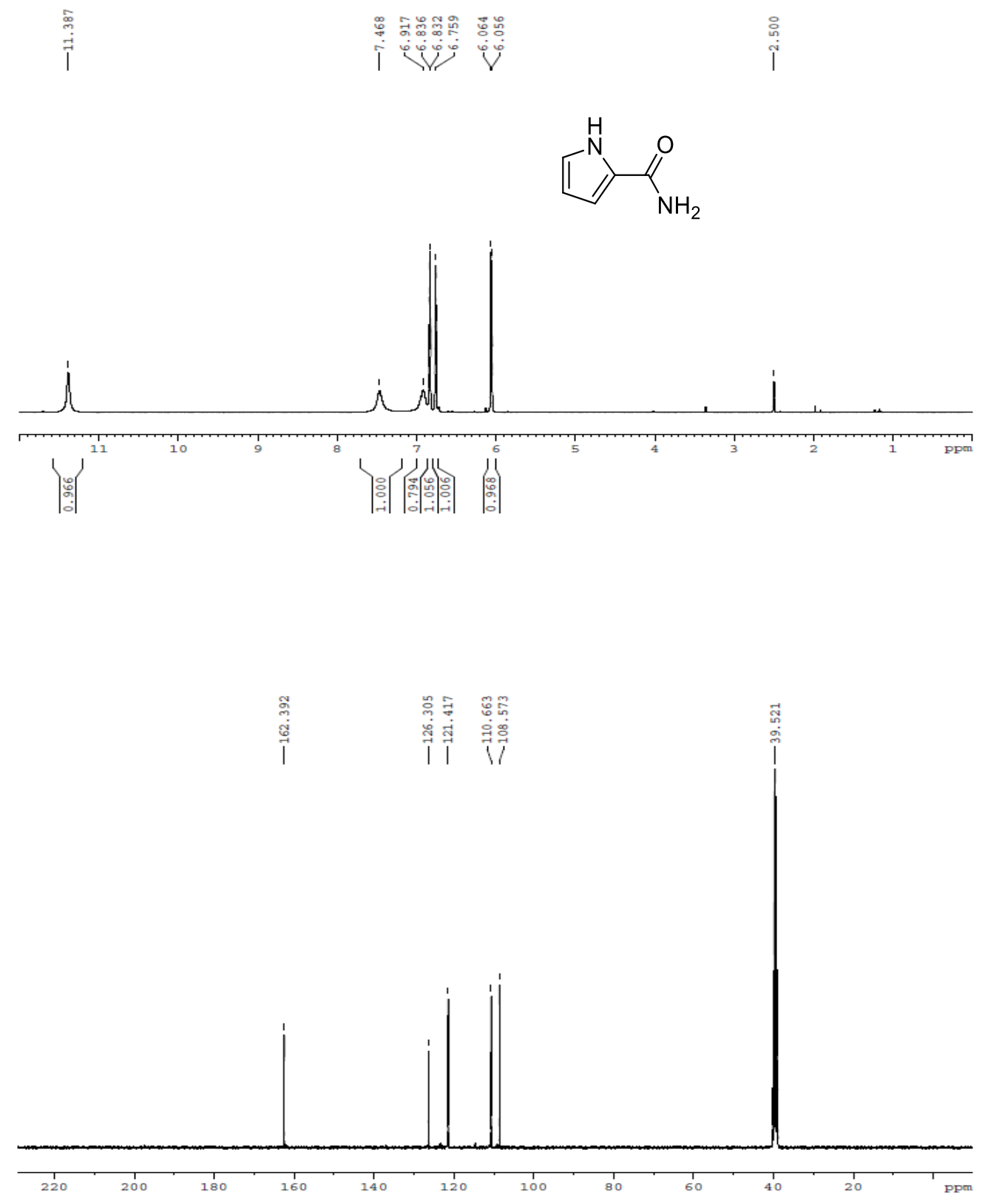


\section{References}

1. R. S. Ramon, N. Marion and S. P. Nolan, Chem. Eur. J. 2009, 15, 8695.

2. A. D.Roy,; A.Subramanian,; R.Roy, J. Org. Chem. 2006, 71, 382.

3. A. Schnyder, M. Beller, G. Mehltretter, T. Nsenda, M. Studer and A. F. Indolese, J. Org. Chem. 2001, 66, 4311.

4. M. A. Ali and T. Punniyamurthya, Adv. Synth. Catal. 2010, 352, 288.

5. R. S. Ramon, J. Bosson, S. Diez-Gonzalez, N. Marion and S. P. Nolan, J. Org. Chem. 2010, 75, 1197.

6. W.-C. Lee and B. J. Frost, Green Chem. 2012, 14, 62. 